\title{
Packing Polymorphism Affecting the Optoelectronic Properties of a $\pi$-Conjugated Organic Compound
}

Gilles H. Roche ${ }^{1,2}$, David Flot ${ }^{3}$, Joël J. E. Moreau ${ }^{1}$, Olivier J. Dautel ${ }^{1}$, Jean-Sébastien Filhol', Arie van der Lee ${ }^{* 5}$,

1 Institut Charles Gerhardt de Montpellier, UMR-5253, Université de Montpellier, ENSCM, CNRS, Montpellier, France, 2. Université de Bordeaux, IMS, CNRS, UMR-5218, Bordeaux INP, ENSCBP, 33405 Talence, France. 3 European Synchrotron Radiation Facility, 71 Avenue des Martyrs, 38000 Grenoble, France 4 Institut Charles Gerhardt de Montpellier, Laboratoire CTMM, UMR-5253, Université de Montpellier 5 Institut Européen des Membranes, IEM - UMR 5635, ENSCM, CNRS, Université de Montpellier, Montpellier, France

KEYWORDS Polymorphism, DFT, NCI, organic semiconductor

ABSTRACT [1]Benzothieno[3,2-b]benzothiophene (BTBT) derivatives are widely employed as
hole transport materials in organic field-effect transistors. The electronic properties of these 
materials depend critically on the crystal packing, which depends on its turn on the choice of the attached functional group. With symmetrically attached alcohol chains the structure may display different packing modes depending on the number of $\mathrm{CH}_{2}$ groups in the alkyl chain. The dipentanol BTBT derivative has two polymorphs I and II, crystallizing from different solvents, with distinct packing modes and consequently different electronic properties. Whereas the conformational changes are very small between I and II and the hydrogen bonding networks in the structures are identical, the adjacent BTBT cores are differently shifted and oriented with respect to each other. It is shown by Density Functional Theory that polymorph I having unfavorable electronic properties is slightly more stable than polymorph II. This is caused by the much more attractive cross stacking between BTBT cores and C5OH chains in I than in II. The stacking in II originates rather from electrostatic interactions between the BTBT cores. The differences and resemblances with the packing modes of the di-butanol and di-hexanol derivatives are discussed. Both polymorphic forms I and II display negative uniaxial thermal expansion, but in different directions with respect to the packing of the molecules.

\section{Synopsis}

The present study describes the packing polymorphism of a benzothienobenzothiophene derivative containing symmetric hydroxy aliphatic chains of which one form is semiconducting and the other not. Density Functional Theory and Non-Covalent Interaction calculations are used to discriminate the two forms. They are also structurally compared with very similar compounds having one methylene spacer more or less in the aliphatic chain.

\section{Introduction}


Polymorphism is a widely occurring phenomenon in crystalline materials. ${ }^{1-7}$ Physicochemical properties of polymorphs can be very different and thus having implications how they interact with their environment. ${ }^{8} 9$ Especially in pharmaceutical compounds the occurrence of polymorphism has been recognized as an inevitable process, which needs to be screened in an early stage of the development of new molecules in order to avoid undesirable effects when administering the drugs. ${ }^{10,11}$ The number of polymorphs is not limited to two, but can be as high as twelve as was recently reported for 5-methyl-2-[(2-nitrophenyl)amino]-3-thiophenecarbonitrile (also named ROY). ${ }^{12-15}$

A recent data mining study of the Cambridge Structural Database ${ }^{16}$ showed that at the time of writing 4573 distinct organic chemical compounds were flagged as 'polymorphic' in the CSD, of which $75 \%$ had more than 1 polymorphic structure described or reported. A remarkable conclusion from this study was that polymorphic entries within the CSD decrease, as a percentage, temporally, reflecting at the same time the huge number of new compounds being discovered and deposited at the CSD. This confirms in some sense McCrone's thesis ${ }^{17}$ that the discovery of polymorphs is positively correlated with the energy and time put into the study of the compound, or in other words, most single crystalline compounds are not investigated in depth anymore whenever the growth of single crystals suitable for X-ray diffraction analysis appears to be successful. Trials with other solvents are not systematically carried out and growth conditions are not systematically varied, which makes the chance of finding polymorphs smaller. Only concomitant polymorphs may escape to this tendency, although the crystallographer pay pick up only the 'best' crystals and 'forget' other tinier or differently shaped crystals. 
Another important finding in the Kersten study ${ }^{16}$ was the relative important propensity of multi-component systems prone to polymorphism. Steed \& Steed $^{18}$ and Clegg ${ }^{19}$ discussed the relationship between so-called Z'>1 structures, i.e. structures with more than one independent molecule in the asymmetric part of the crystallographic unit cell, and the incidence of polymorphism.

Polymorphism is not only important in pharmaceutics, but also in other fields. We focus here on organic $\pi$-conjugated compounds with excellent semi-conducting properties for organic fieldeffect transistor applications. We present a case of a benzothienobenzothiophene (BTBT) based derivative disubstituted by hydroxy aliphatic chains (pentanol) in positions 2 and 7 with two $\begin{array}{lllll}\text { different } & \text { polymorphs } & \text { I } & \text { and }\end{array}$

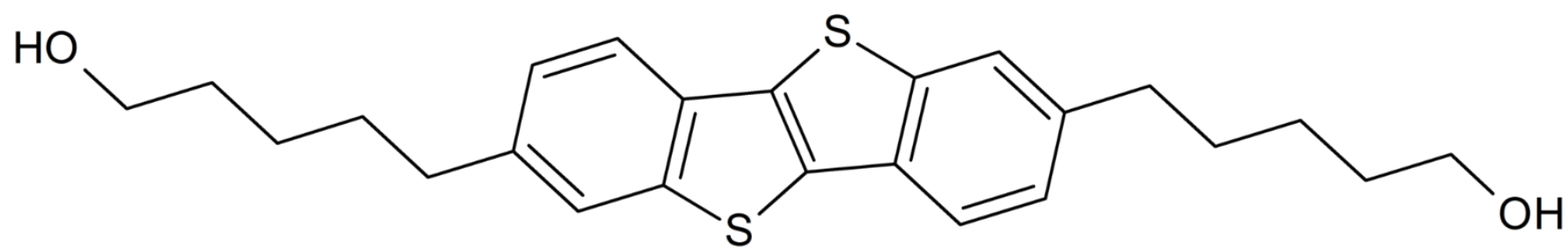

Scheme 1), of which one is semiconducting and the other not. BTBT-C5OH, 5,5'([1]benzothieno[3,2-b][1]benzothiophene-2,7-diyl)bis(pentan-1-ol), is intermediate to BTBT$\mathbf{C 4 O H}^{20}$ and BTBT-C6OH ${ }^{21,22}$ of which we have reported the detailed structural evolution as a function of temperature. These studies showed the existence of isosymmetric phase transitions in both compounds, colossal negative thermal expansion (NTE) in BTBT-C6OH and small NTE in BTBT-C4OH. Using ab initio molecular dynamic studies it was shown that the room temperature structure of BTBT-C6OH is in fact a dynamic mixture of two different phases (but not polymorphs). The structure determinations for BTBT-C5OH were carried out in the framework of a large study on the BTBT-CnOH family of compounds ${ }^{23}$. The structure of II recorded at room 
temperature was reported in that study, but not described in detail. It was noticed as a side remark that the odd members of the series were much more difficult to crystallize than the even members, reflecting also different physical properties for the odd and even members. Based on the relative stacking of the BTBT cores in the structures of the semiconducting $n=6-10$ members a similar stacking was expected for the $n=5$ member. The crystallization in chloroform gave high-quality crystals, but not the expected herringbone packing of the BTBT cores allowing a semiconducting behavior. Therefore, more effort was put into the crystallization of BTBT-C5OH under different conditions and with other solvents. Eventually, with tetrahydrofuran (THF) as solvent, crystals were grown with the same thin plate habit as the $n=6-10$ members and X-ray diffraction analysis gave a similar crystal packing as found for the $n=6-10$ members. While this is a perfect illustration of McCrone's thesis, ${ }^{17}$ it also demonstrates that successful crystal growth is more than serendipity or luck and can be the result of mere perseverance.

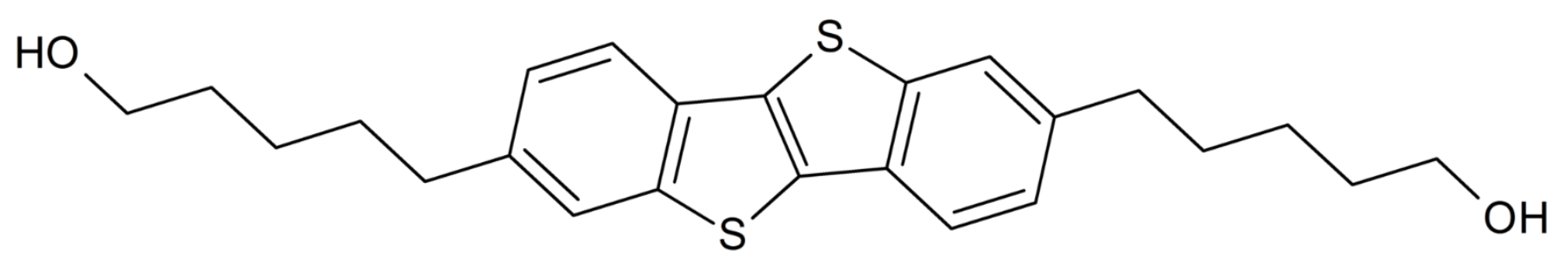

\section{Scheme 1. BTBT-C5OH}

Polymorphism in the field of organic semiconductors can directly affect the desired application, i.e. an as high as possible charge carrier mobility, since the charge transfer integral that depends on the electronic wavefunction overlap is a highly sensitive function of the molecular packing. ${ }^{24-}$

28 This was demonstrated for another BTBT derivative, 2,7-dioctyl[1]benzothieno[3,2$b][1]$ benzothiophene, BTBT-C8, which, when deposited in the form of thin films using a novel 
off-centre spin-coating method, displayed a higher mobility and a different molecular packing than the usual equilibrium structure..$^{29}$

In this study a detailed comparison is given between the structures of the two polymorphs of BTBT-C5OH and those of their $n=4$ and $n=6$ neighbors. Both low-temperature (LT) and close to room temperature (RT) structures are reported for both polymorphs. The Non-Covalent Interactions (NCI) descriptor (14) is used to analyze the packing differences between the polymorphs. Density Functional Theory (DFT) calculations are carried out to quantify the differences between the polymorphs and the charge carriers present.

\section{Methods and materials}

\subsection{Crystal growth}

The synthesis of BTBT-C5OH is reported elsewhere. ${ }^{30}$ Polymorph I was obtained by slow evaporation of a saturated solution in THF disposed in a vial closed by a cap made of cotton at room temperature. Polymorph II was obtained during the cooling of a saturated solutions of BTBT-C5OH in chloroform $\left(\mathrm{CHCl}_{3}\right)$. The solution, in a closed vial, was heated before to be disposed in an oven at $60^{\circ} \mathrm{C}$. Crystals could be obtained during the cooling to room temperature at a rate of $3^{\circ} \mathrm{C}$ per hour. Crystals of $\mathbf{I}$ appeared as colourless thick ( $\sim 50 \mu \mathrm{m}$, Fig. S1) millimetre sized platelets, whereas crystals of II grew also as plates, but much smaller and very thin $(\sim 1 \mu \mathrm{m}$, Fig. S1).

\subsection{X-ray diffraction}

Diffraction data for the crystal structure of polymorph I were collected on a Rigaku-Oxford Diffraction Gemini diffractometer equipped with a Saphire-2 CCD detector with sealed-tube Mo- 
$K \alpha$ radiation at 115 and $275 \mathrm{~K}$. The data collection was set up using CRYSALIS-PRO ${ }^{31}$ and the integration of the data frames was done with the same program using default parameters. Lorentz and polarization effects were also corrected, and the empirical absorption correction was done using spherical harmonics employing symmetry-equivalent and redundant data.

Data for the crystal structure of polymorph II were collected on beamline ID29 of the European Synchrotron Radiation Facility (ESRF) in Grenoble, France, with an ARINAX-MAATEL MD2 diffractometer equipped with a Dectrix Pilatus-6MF detector. Data collection was performed with the MXCUBE program ${ }^{32}$ at 100 and $295 \mathrm{~K}$ and the raw image data were reduced with $X D S^{33,34}$ using default parameters.

The crystal structures were solved and the space group symmetry determined using the ab initio iterative charge flipping method with parameters described elsewhere ${ }^{35}$ using the SUPERFLIP program. ${ }^{36}$ Both structures crystallize in space group $P 2_{1} / c$ and no change of space group was detected with temperature. One of the structures, II, has a $\beta$-angle close to $90^{\circ}$, and a check of possible twinning 37,38 indicated an $80 \%$ probability for pseudo-merohedral twinning and consequently an orthorhombic space group. This hypothesis was ruled out, because of a much higher $R_{\text {int }}$ for orthorhombic symmetry and an unsatisfactorily refinement in the orthorhombic space group.

The structural models were refined against $|F|$ using full-matrix non-linear least-squares procedures as implemented in $C R Y S T A L S^{39}$ on all independent reflections with $I>2 \sigma(I)$. Table S1 gives full details about the data collections and the crystal structure refinements for the four data collections.

\subsection{Ab initio computational methods}

The starting point of the $a b$ initio calculation of the two BTBT-C5OH polymorphs were the experimental LT unit cells containing 224 atoms and four BTBT-C5OH molecules. The 
computation of the properties of very soft materials with a cohesive energy mostly dominated by van der Waals forces requires the use of a functional that includes dispersion contributions and the periodic electronic structure calculations were therefore performed using DFT within the PBE+D3 generalized gradient approximation including van der Waals contributions. ${ }^{40,} 41$ Projected augmented wave (PAW) pseudopotentials ${ }^{42}$ were utilized as implemented in the VASP code. ${ }^{43}$, ${ }^{44} \Gamma$-Centered $(1 \times 2 \times 2)$ and $(3 \times 1 \times 2) k$-point meshes with a high $k$-point density in the direction of the smallest unit cell parameter were employed for the I and II polymorphs, respectively. The structures were computed using a $550 \mathrm{eV}$ cut-off to account for the more electronegative oxygen and sulphur atoms and to properly describe the electronic polarization with the optimization of the positions of all or just a part of the atoms present in the unit cell, and with or without the optimization of the cell parameters. the residual forces on optimized atoms were lower than 0.01 $\mathrm{eV} \AA^{-1}$ for all calculations. The program PLATON ${ }^{37,38}$ was used to recalculate the asymmetric unit content from the full unit cell content output resulting from the DFT calculations using the instruction CALC ADDSYM EXACT followed by ADDSYM SHX. Vibrations within the harmonic approximation were computed using finite displacements of $0.01 \AA$ to derive the dynamic matrix that was diagonalized to extract vibrational frequencies. Finite-temperature corrections and zero-point energies were calculated from these vibrations using a physicalstatistical approach, and in order to obtain the temperature dependent free energy.

\section{Results}

\subsection{Crystal structure descriptions}

Both polymorphs are $Z^{\prime}=(0.5+0.5)$ structures, since they contain both two independent halfmolecules in the asymmetric unit (Figure 1). Each independent molecule has internal inversion symmetry. A complete MOGUL ${ }^{45}$ analysis with CSD 5.41 including the May2020 update ${ }^{46}$ on bond lengths, valence and torsion angles, and ring geometry show two unusual features: the torsion angle C5-C6-C9-C10 in polymorph $\mathbf{I}$ at $115 \mathrm{~K}$ and $275 \mathrm{~K}$ with observed values $132.2(2)^{\circ}$ and 
$134.5(4)^{\circ}$ relative to an approximate mean database value of $90^{\circ}$ for 4955 entries and the C6-C7C8 valence angle for polymorph II at $295 \mathrm{~K}$ is slightly out of range as well with a value of $123.4(6)^{\circ}$ compared to the mean value $121.07^{\circ}$ with standard deviation $1.133^{\circ}$ for 6520 database entries. Fig. S4a-b give the complete histograms resulting from the MOGUL analyses. The deviating torsion angle in $\mathbf{I}$ optimized by DFT at $0 \mathrm{~K}$ is $132.15^{\circ}$ whereas the C6-C7-C8 angle in II is $122.05^{\circ}$. The deviating torsional angle can thus be considered as a genuine structural feature of I, whereas the deviating angle in II is probably due to a slightly less accurate structural model due to the very weak scattering power of this compound.

Interestingly, in both polymorphs the two independent molecules have a different orientation of the hydroxyl group with respect to the $\mathrm{C}_{5} \mathrm{H}_{10}$ alkyl chain: one is cis and the other is trans.

\section{BTBT-C5OH-I}
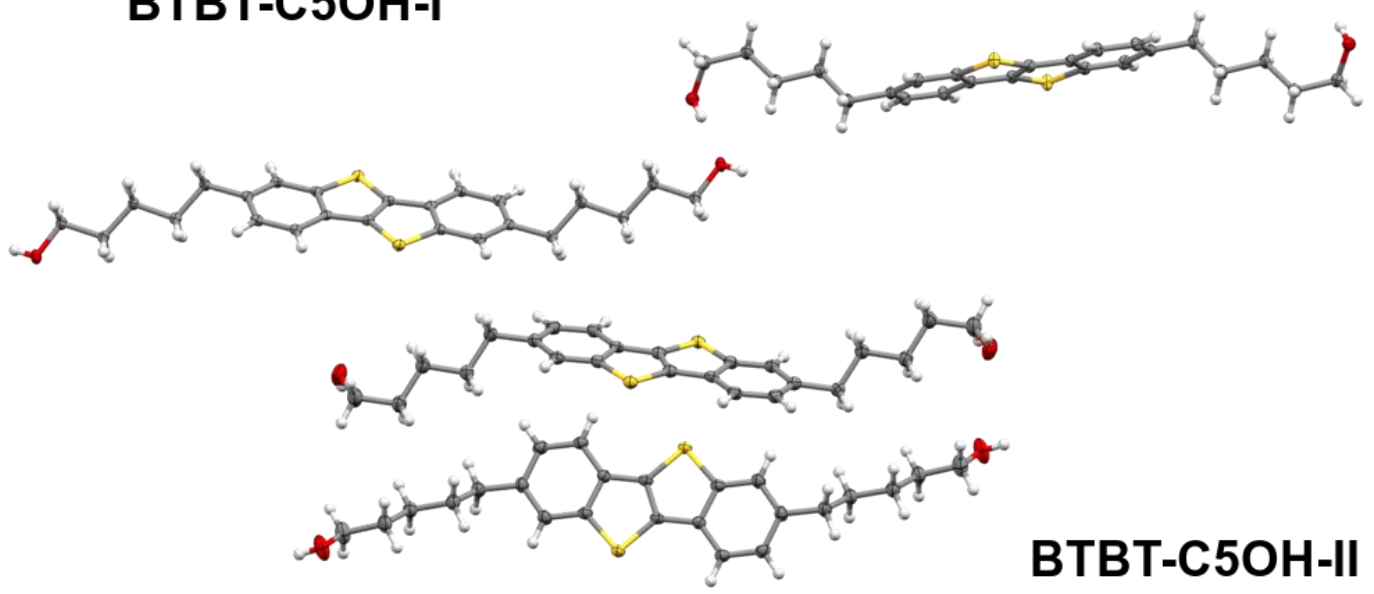

BTBT-C5OH-II

Figure 1. Atomic displacement ('ORTEP') plots of the polymorphs I (115 K) and II (100 K) of the structure of BTBT-C5OH. The upper two molecules represent the structure of $\mathbf{I}$ and the lower two molecules that of II. The atomic displacement ellipsoids are at the $75 \%$ probability level. Note the cis and trans conformations of the hydroxyl groups in the different molecules. All independent half-molecules have been completed by application of the internal inversion symmetry. Atom 
labels are omitted for clarity; detailed atomic displacement plots with atoms labels of the two polymorphic forms are reported in Fig. S3.

The conformational flexibility of the independent molecules within each structure and between the two polymorphs can be assessed by calculating the best molecular overlap between the molecules. Table 1 gives the RMS deviations of the overlapped molecules and maximum distances between the overlapped molecules calculated with MERCURY. ${ }^{47}$ Between the two polymorphs there are four different overlap possibilities and between two temperatures within one polymorph two overlap possibilities.

Table 1. Molecular overlap RMS values and maximum distances between I and II

\begin{tabular}{|c|c|c|l|l|l|}
\hline \multirow{4}{*}{} & \multicolumn{2}{|c|}{ I-II } & & & \\
\cline { 2 - 6 } & rms & max & & rms & max \\
\hline \multirow{4}{*}{ LT } & 0.48 & 0.68 & I & 0.47 & 1.44 \\
\cline { 2 - 6 } & 0.69 & 1.06 & II & 0.57 & 1.84 \\
\cline { 2 - 6 } & 0.87 & 2.33 & & & \\
\cline { 2 - 6 } & 0.81 & 1.44 & & & \\
\hline \multirow{4}{*}{ RT } & 0.71 & 1.07 & I & 0.47 & 1.42 \\
\cline { 2 - 6 } & 0.80 & 1.42 & II & 0.55 & 1.78 \\
\cline { 2 - 6 } & 0.49 & 0.70 & & & \\
\cline { 2 - 6 } & 0.84 & 2.28 & & & \\
\hline
\end{tabular}

Notes: All values are in $\AA$. The values are given for the structures at low temperatures (LT) and close to room temperature (RT). 
The results in Table 1 show that the polymorphism is not of conformational nature and thus rather of the packing type. Within each phase the conformational changes with temperature are also rather small.

\subsection{Crystal structure packings}

Figure 2 shows the different packings of the structures of I and II. 
(a)
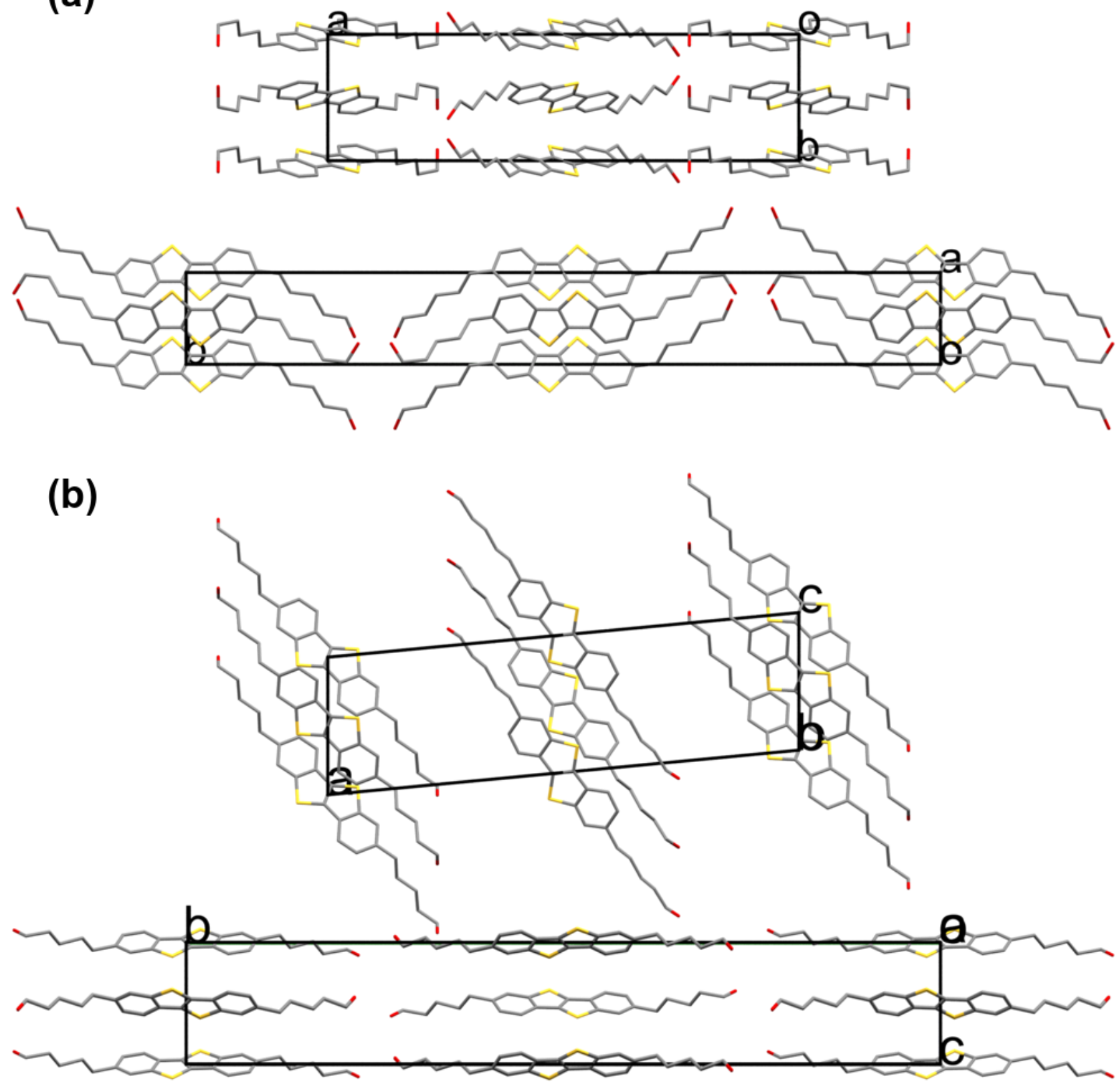

Figure 2. Crystal packings of structures I and II at low temperatures. The upper structure in each substructure is I and the lower II. The scale in each subfigure (a) and (b) is the same, but there is a slight scale difference between (a) and (b). The subfigure (b) was obtained from (a) by a $90^{\circ}$ rotation about the horizontal $x$-axis of each polymorph. 
The molecules of both polymorphs pack in layers perpendicular to the $a^{*}$ axis for $\mathbf{I}$ and perpendicular to the $b^{*}$ axis for II. The layers are connected via hydrogen bonds between the hydroxy-alkyl chains of different layers. The molecular arrangement within the layers is, however, completely different. In the structure of II the BTBT cores are spatially aligned and non-shifted in such a way that close intermolecular S $\cdots$ S contacts exist of $3.619 \AA$ at $100 \mathrm{~K}$ and $3.656 \AA$ at 295 $\mathrm{K}$ which is only slightly larger than twice the van der Waals radius $\left(1.80 \AA{ }^{48}\right)$ of sulphur. In the structure of I on the other hand, these close S $\cdots S$ contacts are completely absent, since the BTBT cores of neighbouring molecules are laterally shifted along the axes of the BTBT cores. The interactions between the neighbouring molecules in I are mainly of the $\mathrm{CH} \cdots \pi$ type.

The orthogonal thermal expansion coefficients $\mathrm{X} 1, \mathrm{X} 2, \mathrm{X} 3$, and $\propto_{V}$ calculated from the cell parameters at low temperatures and near ambient temperatures have been collected in Table 2. The thermal Indicatrix Anisotropy Coefficients (IACs; ${ }^{20,49}$ ), which are a measure - between -1 and $1-$ of the anisotropy of the thermal expansion, have also been calculated.

Table 2. Thermal expansion properties of the two polymorphs of BTBT-C5OH.

\begin{tabular}{|l|l|l|l|l|l|c|}
\hline & $\mathrm{X} 1$ & $\mathrm{X} 2$ & $\mathrm{X} 3$ & $\propto_{V}$ & $\mathrm{IAC}$ & $\Delta \rho$ \\
\hline $\mathbf{I}$ & -38.34 & 52.90 & 152.27 & 167.13 & $0.38(-++)$ & -2.6 \\
\hline II & 44.07 & -48.56 & 125.29 & 120.27 & $0.52(+-+)$ & -2.3 \\
\hline
\end{tabular}

Notes: the values for the thermal expansion coefficients X1, X2, X3 and $\propto_{V}$ are in $\mathrm{MK}^{-1}$, where $\mathrm{X} 1, \mathrm{X} 2, \mathrm{X} 3$ are the three orthogonal principal axes of the thermal expansion tensor for the axes, and $\propto_{V}$ the volumetric thermal expansion coefficients. X1, X2, and X3 are increasingly ordered by their absolute values; their sign is indicated within the parentheses following the IAC value. $\Delta \rho(\%)$ is the decrease of the density between the lowest and highest measurement temperature. 
It is interesting to note that both polymorphs display uniaxial negative thermal expansion of nearly equal magnitude: I within the $a c$-plane and II perpendicular to the $a c$-plane, which in terms of the molecular packing means that the negative thermal expansion in $\mathbf{I}$ is within the molecular planes, and in the case of II perpendicular to them. The cell parameters of I were also measured at intermediate temperatures and confirm the existence of the uniaxial negative thermal expansion (Fig. S2). The cell parameters of both structures were optimized at $0 \mathrm{~K}$ (see DFT section): the expansion along the axes which display negative uniaxial thermal expansion between $100 \mathrm{~K}$ and room temperature, show normal positive thermal expansion between $0 \mathrm{~K}$ and $100 \mathrm{~K}$. This could indicate a crossover between a positive thermal expansion at low temperatures and negative thermal expansion at higher temperatures as is found for BTBT-C4OH. ${ }^{20}$

Polymorph II is significantly less dense than polymorph I: $1.278 \mathrm{gcm}^{-3}$ versus $1.314 \mathrm{gcm}^{-3}$ near room temperature for I and II, respectively. This would imply, according to the well-known Burger \& Ramberger 'density rule', ${ }^{50}$ that polymorph I having the 'best' packing should have the lowest free energy at absolute zero, but it was shown in a recent study that this density rule is violated for almost half of the investigated monotropic cases,${ }^{51}$ contrary to Burger \& Ramberger's $10 \%$ claim.

\subsection{DFT structures}

The first step in the DFT calculations performed at $0 \mathrm{~K}$ was to relax the positions hydrogen atoms while keeping the other atoms fixed as well as the cell parameters. The experimental X-ray structures of I and II at $115 \mathrm{~K}$ and $100 \mathrm{~K}$, respectively, were used as starting points $\mathbf{O} 0$ for the first optimisation $\mathbf{O} 1$. This led to $\mathrm{C}-\mathrm{H}$ and $\mathrm{O}-\mathrm{H}$ bond lengths very close to their neutron values of 1.089 and $0.993 \AA$, respectively, accompanied by a H-relaxation of about $300 \mathrm{meV}$ per hydrogen and 36 
eV per unit cell. Then all atoms were relaxed but the cell parameters were kept fixed (O2) and finally the cell parameters were also relaxed (O3). Table 3 gathers the results of the optimizations $\mathbf{O} 0, \mathbf{O} 1, \mathbf{O} 2$, and $\mathbf{O} 3$, where $\mathbf{O} 0$ is the starting point, i.e. the $\mathrm{X}$-ray structures.

The computed energy changes from $\mathbf{O} 1$ to $\mathbf{O} 2$ for both polymorphs are lower than $200 \mathrm{meV}$ per unit cell, i.e. less than $10 \mathrm{~J} . \mathrm{mol}^{-1} /$ atom. This weak energy change already suggests that the calculated structures remains close to the experimental ones. The full unit cell optimization $(\mathbf{O} 2$ to O3) leads to even weaker energy changes less than $30 \mathrm{meV} / \mathrm{unit}$ cell, i.e. $1.5 \mathrm{Jmol}^{-1} /$ atom. These changes are in the expected range for phenomena linked to thermal expansion.

These calculations strongly suggest that polymorph I is slightly more stable than polymorph II, in agreement with the not so trustworthy Burger \& Ramberger density rule. ${ }^{50,51}$ Their relative energies are nevertheless very close with less than $1 \mathrm{meV}$ per atom.

Table 3. Energies of the DFT optimizations $\mathbf{O} 0 \cdots \mathbf{O} 3$

$\begin{array}{cccccccc} & \mathrm{O} 0 & \mathrm{O} 1 & \mathrm{O} 2 & \mathrm{O} 3 & O 3+Z P E & V(\mathrm{O} 3) & V(\mathrm{O} 0) \\ \mathrm{I} & - & - & -1380.83 & -1380.85 & -1330.61 & 2047.57 & 2086.34 \\ & 1344.35 & 1380.64 & & & & & \\ \mathrm{II} & - & - & -1380.69 & -1380.71 & -1330.53 & 2112.97 & 2144.71 \\ & 1343.93 & 1380.58 & & & & & \\ & -0.42 & -0.06 & -0.14 & -0.14 & -0.08 & -65.40 & -58.37\end{array}$

Notes: See the main text for a definition of the different optimizations $\mathbf{O} 0 \ldots \mathbf{O} 3+\mathrm{ZPE}$ (zero point energy). The energies are in $\mathrm{eV} . V(\mathbf{O} 3)$ and $V(\mathbf{O} 0)$ are the cell volumes of the final optimizations and of the X-ray structures in $\AA^{3}$, respectively.

The DFT optimized structures were compared with the LT experimental structures via different structure similarity indicators, viz. the root mean square Cartesian displacement (RMSC, $\left.{ }^{52}\right)$, the mean difference distance $<\delta>$ between similar atoms and the measure of similarity $\Delta$, defined by Bergerhoff et al. ${ }^{53}$ and calculated with COMPSTRU ${ }^{54}$ which performs the analysis in real space, 
and the structure similarity parameter $\phi_{\text {struc }}$ defined in the SUPERFLIP program ${ }^{36}$ which is rather a measure of similarity in reciprocal space since it measures the similarity between the structure factors calculated from the atomic structures.

Table 4. Similarity between DFT optimized structures (O3) and experimental low temperature structures.

\begin{tabular}{clllll}
\hline & RMSCD1 & RMSCD2 & $<\delta>$ & $\Delta$ & $\phi_{\text {struc }}$ \\
O3_I(0 K)-I(115 K) & 0.044 & 0.114 & 0.095 & 0.015 & 0.0050 \\
\hline O3_II(0 K)-II(100 K) & 0.035 & 0.110 & 0.089 & 0.028 & 0.0036 \\
\hline I(115 K)-I(275 K) & 0.051 & 0.093 & 0.078 & 0.053 & 0.0038 \\
II(100 K)-II(295 K) & 0.073 & 0.124 & 0.089 & 0.018 & 0.0169
\end{tabular}

Notes: See for a definition of the different similarity indices the main text. The similarity indicators between the low temperature structures and the room temperature structures for each polymorph are given in the two lower lines for comparison. Hydrogen atoms are included in the calculations in all cases, except for RMSCD1. The positions of the hydrogen atoms of the DFT structures have not been reset to their experimental X-ray positions.

Since the three parameters measure different similarities, it is not expected that they should give the same trend for the four different comparisons (Table 4). The purely geometric parameter $<\delta>$ is fairly constant, whereas $\Delta$, which takes into account as well the spontaneous lattice strain, shows more disperse values. Interestingly, the parameter $\phi_{\text {struc }}$ which measures the similarity in Fourier space, displays a rather deviating value for the diffraction patterns of the LT and RT phases of II without any obvious reason. Van de Streek \& Neumann ${ }^{52}$ showed that RMSCD values for molecular single-crystal structures calculated without $\mathrm{H}$ atoms below $0.25 \AA$ indicate in general 'correct' experimental structures. From the comparison with the RMSCD values calculated between the LT and RT experimental structures it may be deduced that the RMSCD values calculated between the $0 \mathrm{~K}$ DFT structures and the LT structures include a more or less important 
temperature dependent component. The main conclusion from this comparison is that the experimental structures are without any doubt correct and that the DFT structures are a faithful representation of the experimental structures extrapolated to $0 \mathrm{~K}$. Therefore, the DFT structures can be used to investigate the different BTBT-C5OH's moieties contributions to the total crystal cohesive energy in the two different polymorphic structures.

\subsection{Decomposition of the polymorph's cohesive energies}

Because of the very small energy difference between the two polymorphs (less than 14 $\mathrm{kJ} / \mathrm{mol} /$ unit cell), zero-point energies (ZPE) were computed from phonons and included in the energies of $\mathrm{O} 3$ (see Table 3). The energy difference between the two polymorphs decreases even more to only $7.7 \mathrm{~kJ} / \mathrm{mol} /$ unit cell (i.e. less than $2 \mathrm{~kJ} / \mathrm{mol}$ per molecule). The reduction in the energy difference is caused by polymorph I having slightly higher O-H frequency that II (e.g. the highest $\mathrm{OH}$ stretch frequency is $3287 \mathrm{~cm}^{-1}$ for I while it is $3149 \mathrm{~cm}^{-1}$ for II). This is consistent with the OH distance of $1.00 \AA$ in I and $1.01 \AA$ in II. This also suggests that the intermolecular hydrogen bonds are slightly more strained and weaker in I than in II.

In order to investigate the different contributions to the total cohesive energy, the BTBT-C5OH molecule was optimized alone in a unit cell large enough to remove all the intermolecular interactions in order to obtain the cohesive energy (including the zero-point energy) of the free BTBT-C5OH molecule inside the structures of the two polymorphs. In this way the cohesive energy of the molecule (including zero-point energy effects) in the crystal can be computed to be -244.9 and $-242.8 \mathrm{~kJ} \mathrm{~mol}^{-1}$ in the I and II polymorphs, respectively. Polymorph I appears to be

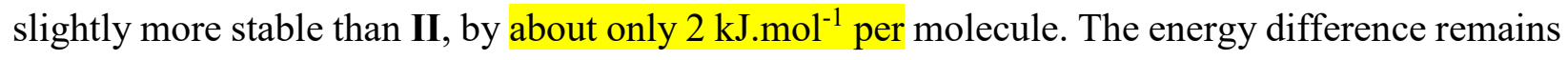
very low as can be expected for (kinetically) stable polymorphic structures. 
In order to better understand the different energy contributions to the cohesive energy, we have partitioned the intermolecular interactions into three terms: the BTBT/BTBT intermolecular stacking interactions, the intermolecular $\mathbf{C 5 O H} / \mathbf{C 5 O H}$ stacking interactions and the intermolecular cross-interactions between a lateral C5OH and BTBT cores. To obtain this decomposition, the BTBT-C5OH molecule is split into independent BTBT and C5OH subunits each of them saturated with hydrogens to compensate the broken $\mathrm{C}-\mathrm{C}$ bond. Then, these subunits are separately computed in the frozen geometry of the I and II polymorphic unit cell with only the added saturating hydrogens being optimized. The crystals with only BTBT/BTBT and $\mathbf{C 5 O H} / \mathbf{C 5 O H}$ subunits in the I and II structure are obtained and the associated interaction energy computed. Then, the different inter-moieties interaction energies are extracted as is shown schematically in Figure 3. In the two polymorphs, the $\mathbf{C 5 O H} / \mathbf{C 5 O H}$ interaction is the main contribution ensuring about $50 \%$ of the cohesive energy as it includes the strong intermolecular H-bonding of the alcohol's moieties. Nevertheless, between I and II, the interaction contributions are quite different. In polymorph II the BTBT/BTBT cores stacking is $25 \%$ stronger than in the structure of BTBT-C5OH-I and the $\mathbf{C 5 O H} / \mathbf{C 5 O H}$ stacking $16 \%$. However, the cross stacking between BTBT cores and $\mathbf{C 5 O H}$ chains is three times more stable in I than in II. Therefore, in I, the softer coupling between adjacent BTBT cores and adjacent $\mathbf{C 5 O H}$ chains than that in II more than fully compensated by the cross-stacking between BTBT and C5OH chains which gives an overall slightly more stable structure for I than for II . 


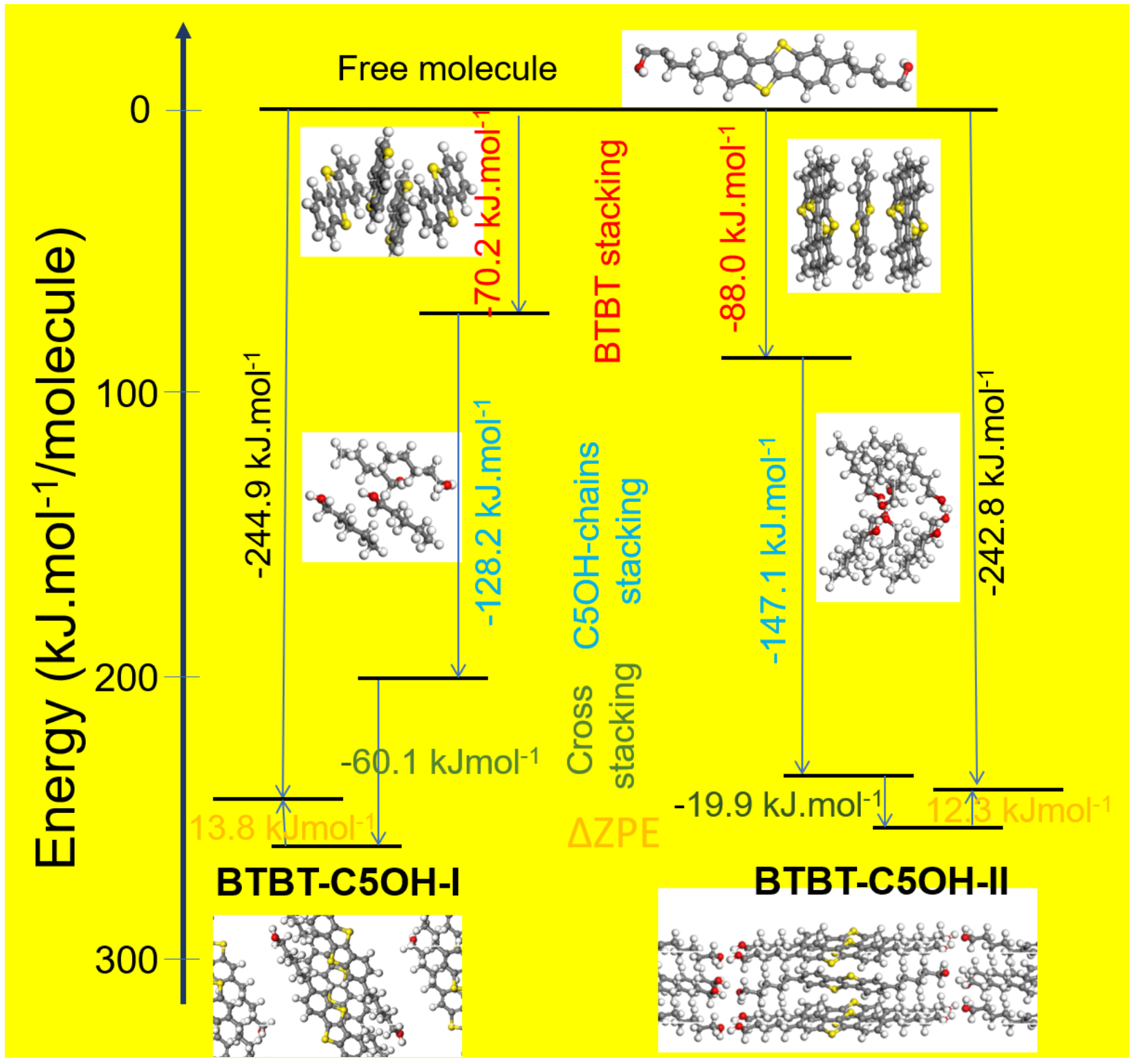

Figure 3. Decomposition of the different energy contributions to the total cohesive energy of the BTBT-C5OH molecule in the crystalline structures of I and II. The red values give an evaluation of the BTBT/BTBT intermolecular interaction energy, the blue ones the $\mathbf{C 5 O H} / \mathbf{C 5 O H}$ intermolecular interaction, the green ones the cross-stacking energy between C5OH and BTBT moieties. The yellow values give the zero-point energies (ZPE) variation contribution to the cohesive energy. The black values give the crystal formation energy from BTBT-C5OH for the two different polymorphs I and II. 


\subsection{DFT computed electronic properties}

The difference in molecular stacking in the I and II polymorphs is expected to modify the electronic properties of the two crystals. The electronic band structures of I and II were computed Fig. 4 and are typical of direct semiconductors with a (DFT) band gap of about $2.2 \mathrm{eV}$. In each case, the lower conduction band and upper valence band are built from the Lowest Unoccupied Molecular Orbitals (LUMO) and Highest Occupied Molecular Orbitals (HOMO), respectively, of the four BTBT-C5OH molecules in the unit cell, without any band crossing. As seen in Fig. 4, most of the HOMO and LUMO orbitals of the BTBT-C5OH molecules are mostly developed on the $\pi$-system of the BTBT moieties with a small contribution of the orbital of the closest $\mathrm{CH}_{2}$ groups that is associated with some classical electron donation/hyperconjugation behaviour of the aliphatic chains toward the BTBT moieties. The electron density variation from isolated subunits (Fig. S7) confirms that there is a charge transfer from the closest $\mathrm{CH}_{2}$ groups in $\mathbf{C 5 O H}$ chains towards the BTBT cores associated to a donation/hyperconjugation effect. In the BTBT cores not all atoms are electron enriched: the 6 closer carbon atoms to the $\mathbf{C 5 O H}$ chains are enriched while other are even depleted (Fig. S7). The BTBT-C5OH molecule presents a strong electrostatic quadrupole with the $\mathrm{CH}_{2}$ methylene group which is closest to the BTBT moieties being positively charged while the 6 aromatic carbon atoms that are within the BTBT core and closest to the chain are negatively charged. Therefore, dipole-dipole van der Waals interactions are expected to be quite important in the cohesive energy of these molecular crystals. Interestingly, in polymorph I, sliding of the relative packing compared to II allows a closer interaction between the negatively 
charge atoms of the BTBT and the positively charged $\mathrm{CH}_{2}$ groups leading to strong cross interactions that stabilizes I relative to II as found above.
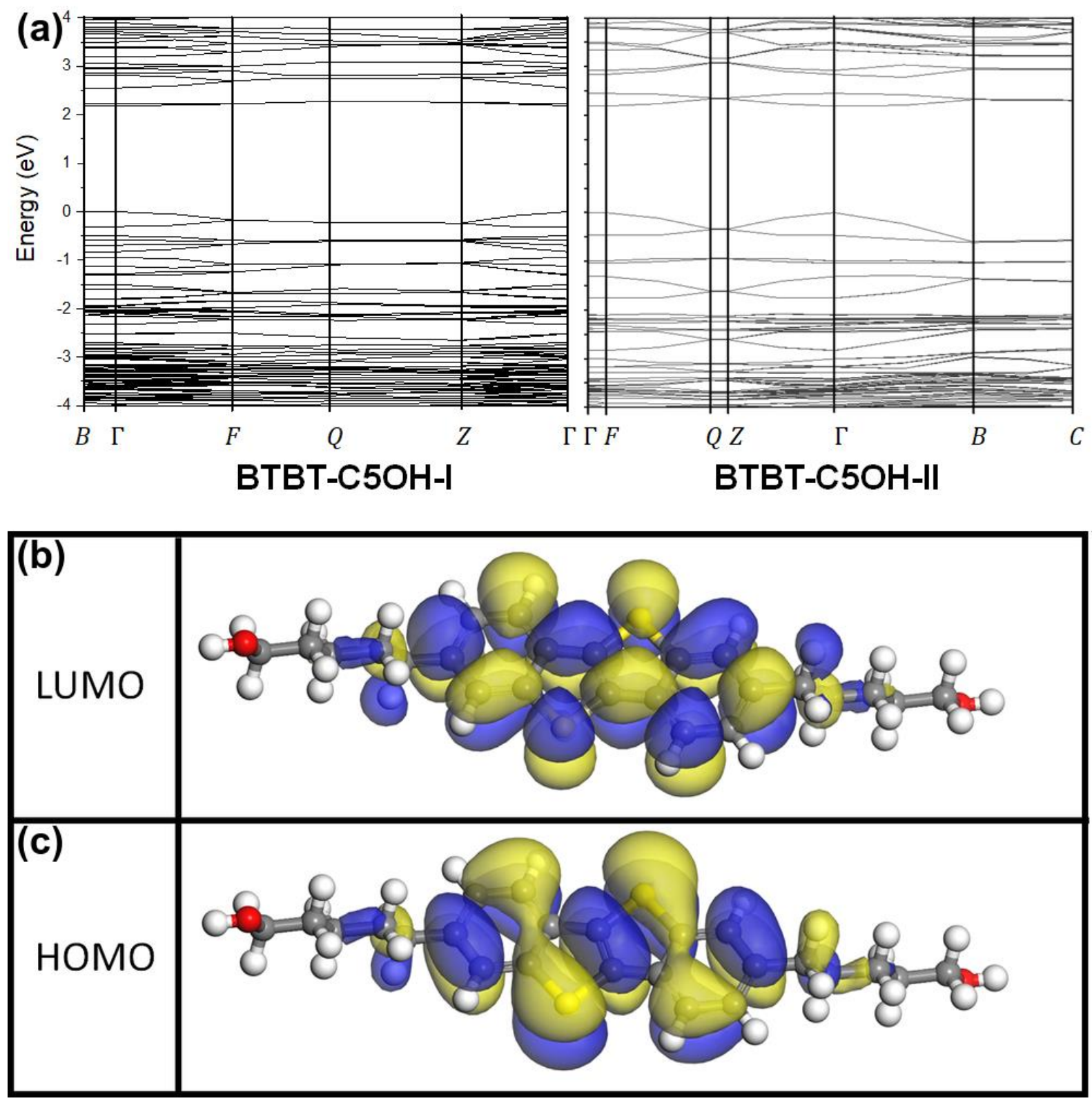

Figure 4. (a) Computed band structures of the BTBT-C5OH-I and BTBT-C5OH-II structures. (b) LUMO and (c) HOMO orbitals of the BTBT-C5OH-II molecule. 
The band structure clearly shows that some partial delocalization exists in the directions perpendicular to the $\mathbf{C 5 O H}$ chains mostly in the plane formed by the BTBT core. The band dispersion is more important for the polymorph II than for the I. This strongly suggests a far better molecular orbital overlap in the case of II, which is fully consistent with the structure of II where the BTBT cores are closely packed according to a herringbone mode. The Fukui functions $\mathrm{f}^{\mathrm{a}}$ and $\mathrm{f}^{+}$that give insight in the real space delocalization of holes and electrons in the valence and conduction bands, respectively for polymorph II are presented in Figure 5. These functions show that electrons and holes are delocalized all over the BTBT core in the same molecular plane and they suggest that this polymorph is a decent organic semi-conductor with a good electron/hole mobility, as is indeed observed experimentally. ${ }^{30}$

For I, as seen from the band structure (Figure 4a), the BTBT cores overlaps are not as strong because of the sliding of the molecule in the crystal, reducing the conjugation and thus electron/hole mobilities.

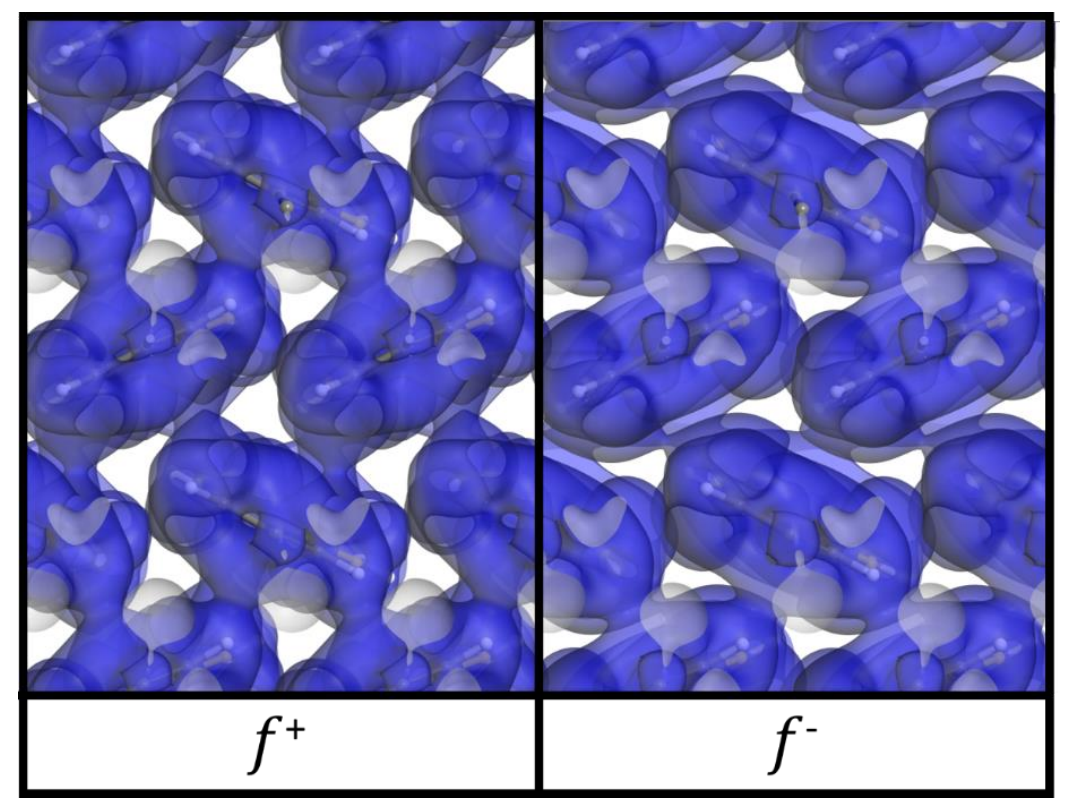


Figure 5. Positive contribution of the Fukui functions $\mathrm{f}^{+}$and $\mathrm{f}^{-}$associated with the hole and the electron density in the conduction and valence bands respectively, for the BTBT-C5OH-II polymorph at the dibenzothiophene moieties.

\subsection{NCI calculations}

The Non-Covalent Interactions (NCI) method uses the scalar electron density function $\rho$ derived from the atomic positions or from quantum-mechanical calculations to determine the reduced density gradient (RDG, $s(\rho)$ ) scalar field (12). Real space is in this way partitioned in different bonding regions depending on the values of $\rho$ and $s$ : low-s high- $\rho$ indicate covalent bonds, low-s low- $\rho$ to non-covalent interactions non-covalent interactions whereas high-s low- $\rho$ correspond to non-interacting density tails. The sign of the second eigenvalue of the density Hessian matrix $\left(\lambda_{2}\right)$ indicates whether the interactions are repulsive or attractive, where negative eigenvalues point to enhanced electron density close to the intermolecular bond critical points and positive eigenvalues represent steric clashes or non-bonding interactions. 

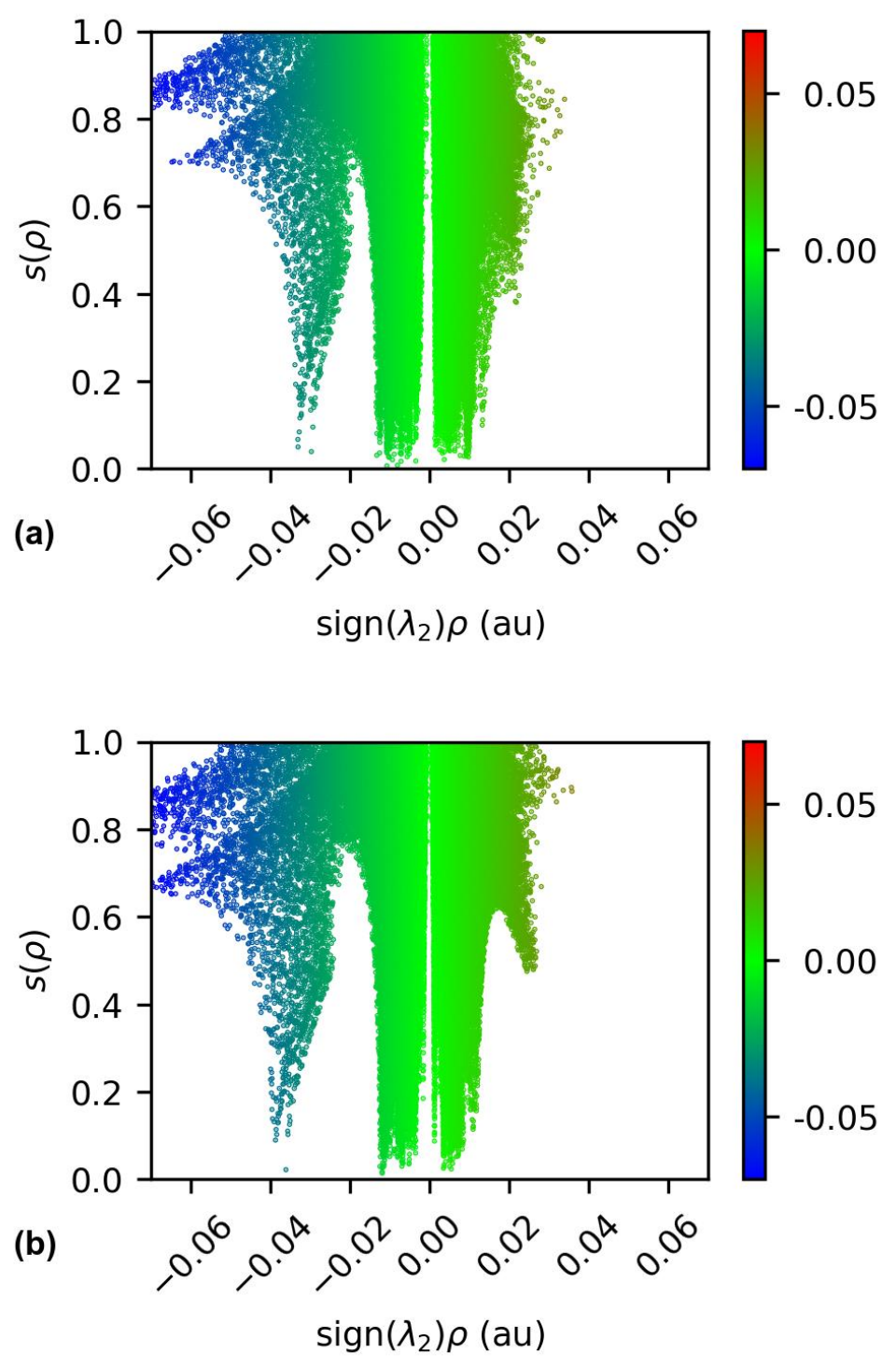

Figure 6. Reduced gradient $(s)$ plots versus the promolecular density multiplied by the sign of the second eigenvalue of the density Hessian matrix $\left(\operatorname{sign}\left(\lambda_{2}\right) \rho\right)$ for polymorph $\mathbf{I}(115 \mathrm{~K}$; a) and polymorph II (100 K; b). A simple colouring scheme based on the value of $\operatorname{sign}\left(\lambda_{2}\right) \rho$ was taken, where blue represent attractive interactions $(-0.07 \mathrm{au})$, green weak Van der Waals interactions $(0.00 \mathrm{au})$, and red repulsive interactions $(0.07 \mathrm{au})$.

Figure 6 gives $s(\rho)$ versus $\operatorname{sign}\left(\lambda_{2}\right) \rho$ for the two polymorphs calculated from the promolecular densities, in which only intermolecular interactions were taken into account using the default 
distinctive parameter $\gamma_{\mathrm{ref}}=0.95$. In both cases a cluster of six molecules (see Fig. S5) was taken for the caluclations. Each cluster contains exactly $5 \mathrm{H} \cdots \mathrm{OH}$ interaction and four diffuse Van der Waals molecules. While the general features are rather similar, they do differ in the details. The blue hydrogen-bonding interactions appear to be slightly stronger in the case of polymorph II than in polymorph I, whereas the green weak Van der Waals interactions are also slightly more developed, especially on the positive side of $\operatorname{sign}\left(\lambda_{2}\right)$.

The NCI regions can be quantified in terms of surface, area, and included charge. Boto et al. (12b) showed in particular that the integrals of the $n=2$, and $n=2.5$ powers of the charge over the NCI regions are highly positively correlated with the interaction energies determined from highlevel DFT calculations. We determined here the NCI qualifiers for the different interaction regimes, i.e. the hydrogen bonding network for $\operatorname{sign}\left(\lambda_{2}\right) \rho$ between -0.1 and -0.02 au, for the Van der Waals interactions between the alkyl chains and the BTBT moieties between -0.02 and 0.02 $\mathrm{au}$, and for the repulsive interactions between 0.02 and 0.1 (nearly absent). Table 5 gives the relevant NCI properties for the two polymorphs within the different $\operatorname{sign}\left(\lambda_{2}\right) \rho$ ranges. They show notably that both the volume of the NCI region and the integrated charge of $\mathbf{I}$ are significantly larger than for the less stable polymorph II. We also used the wave functions determined in the DFT calculations for a full periodic NCI calculation using the program CRITIC-2 (Fig. S6), ${ }^{55}$ but since they did not give additional insight compared to the promolecular cluster calculations, we do not detail them further.

Table 5: NCI indicators for the structures of polymorphs I and II

\begin{tabular}{|c|l|l|l|l|}
\hline & \multicolumn{2}{|l|}{ Polymorph I (115 K) } & \multicolumn{2}{l|}{ Polymorph II (100 K) } \\
\hline $\operatorname{sign}\left(\lambda_{2}\right) \rho(\mathrm{au})$ & $I_{0}$ & $I_{1}$ & $I_{0}$ & $I_{1}$ \\
\hline
\end{tabular}




\begin{tabular}{|l|l|l|l|l|}
\hline$-0.1->0.02$ & 37.1 & 1.34 & 36.2 & 1.44 \\
\hline$-0.02->0.02$ & 1464.0 & 10.0 & 1157.6 & 8.3 \\
\hline $0.02->0.1$ & 2.9 & 0.08 & 1.9 & 0.05 \\
\hline all & 1619.3 & 13.9 & 1288.8 & 11.9 \\
\hline
\end{tabular}

Notes: $I_{0}$ is the volume $\Omega$ of the NCI region, $I_{1}$ is the integrated charge over the NCI volume $\Omega$.

In order to determine spatially which Van der Waals interactions are the most attractive, the reduced gradient density isosurfaces were calculated for the Van der Waals interactions for $\operatorname{sign}\left(\lambda_{2}\right) \rho$ values between -0.02 and 0.02 au (Fig. 7) at the 0.3 RDG isosurface level. 


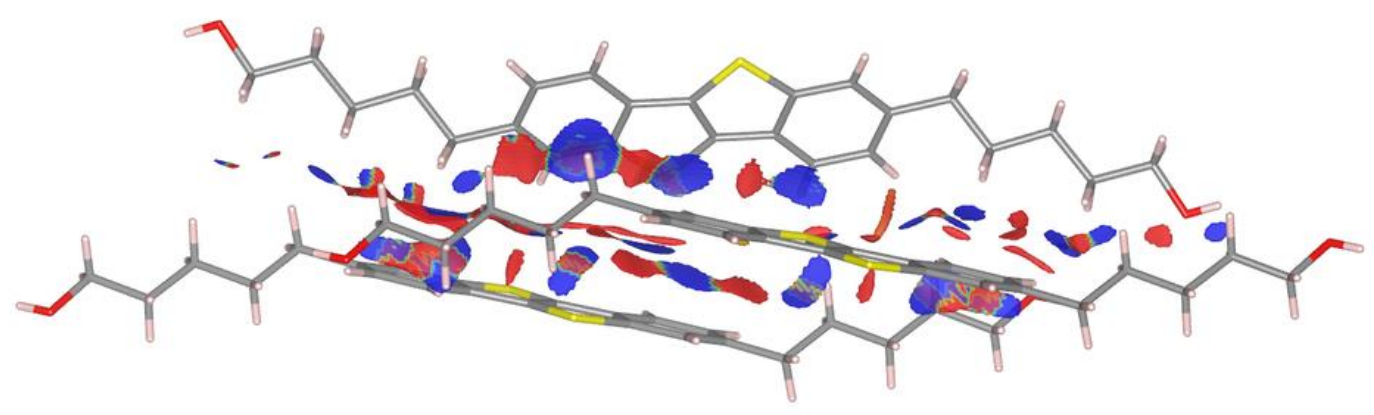

BTBT-C5OH-I

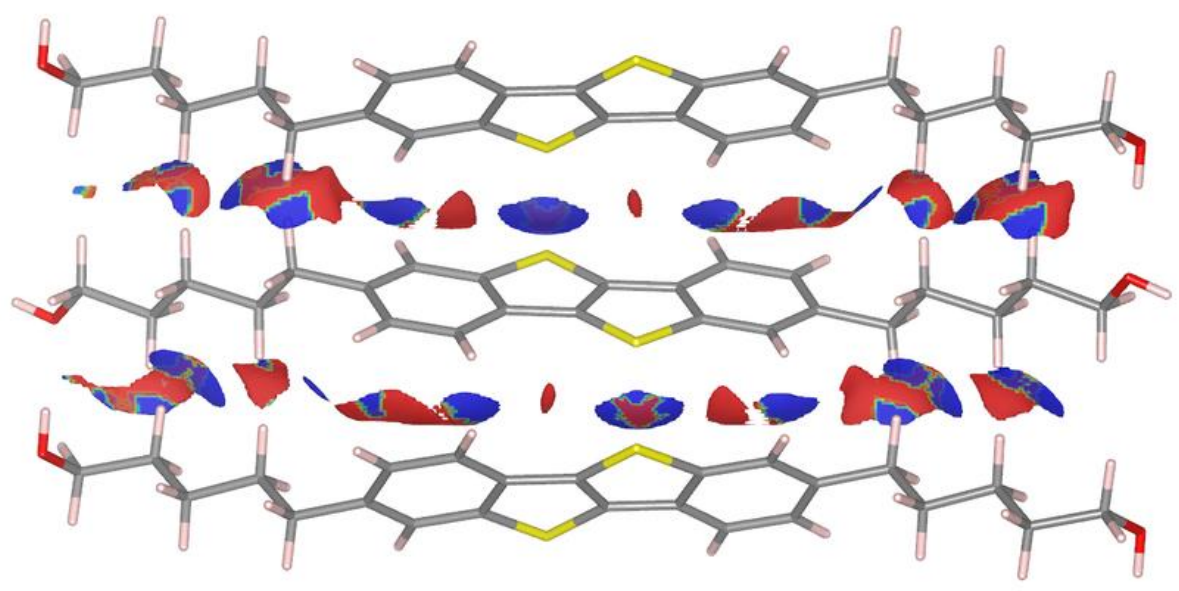

BTBT-C5OH-II

Figure 7. NCI volumes at the isosurface level $\rho=0.3$ au. Upper figure: polymorph I $(115 \mathrm{~K})$; lower figure: polymorph II. The voxel distance used for the calculations is 0.075 au in all three directions. The following colour code based of $\operatorname{sign}\left(\lambda_{2}\right) \rho$ was used: -0.02 au (blue) to 0.02 au (red). Note that this is not the same colour scheme as in Figure 3.

Figure 7 shows the NCI Van der Waals regions between trimers of BTBT-C5OH in the polymorphs I and II. The colour scheme goes from slightly attractive (blue) to slightly repulsive (red). The cohesive interaction in I seems to originate mainly from $\mathrm{CH}^{\cdots} \pi \pi$ interactions between the aliphatic chains from one molecule and the aromatic BTBT core of an adjacent molecule, 
whereas in II it is evenly composed from the contributions between thiophene moieties, $\pi \cdots \pi$ interactions between phenyls and interactions between aliphatic chains of adjacent molecules. This NCI interpretation is fully consistent with the energy decomposition obtained by DFT in the previous section.

\subsection{Finite temperature effect}

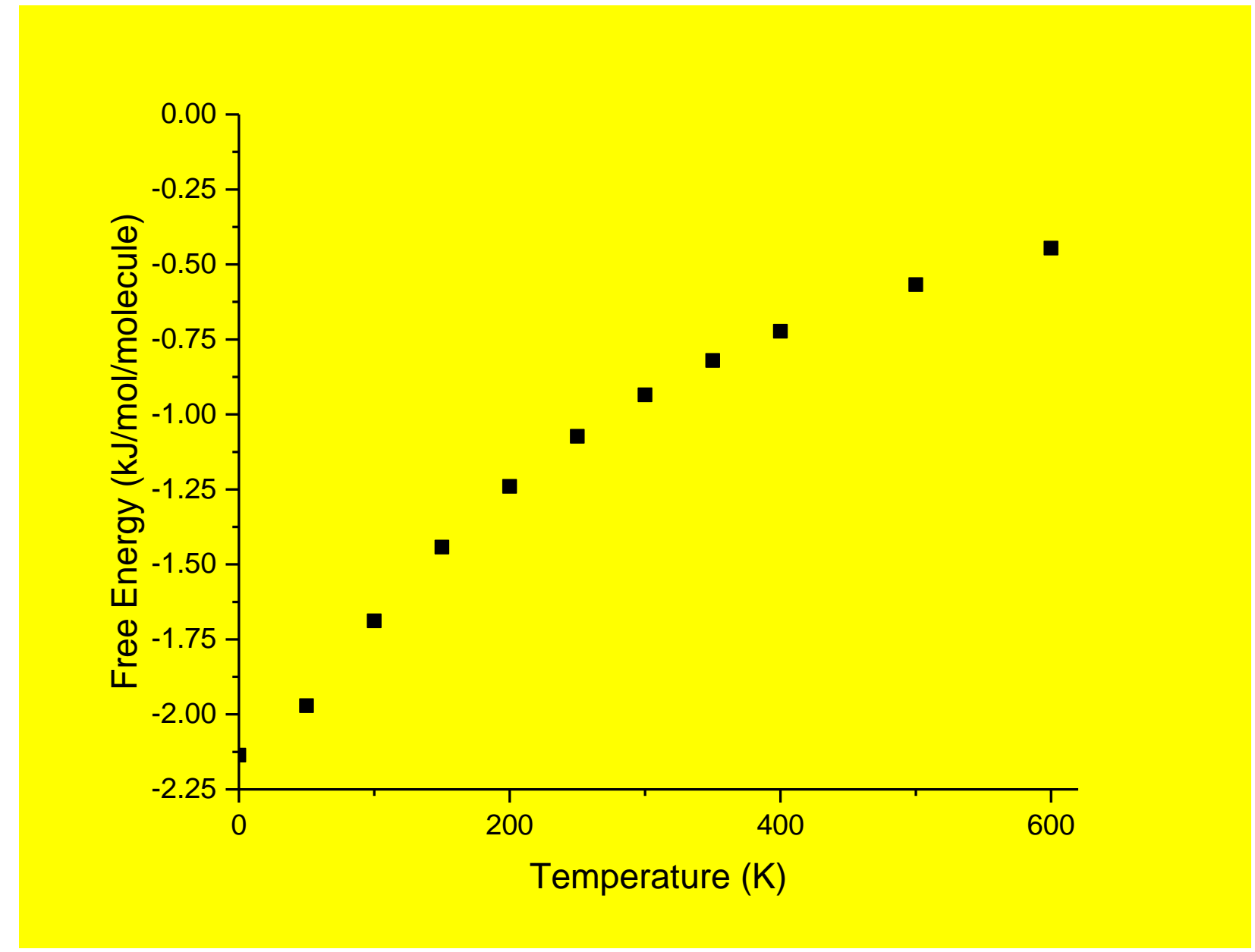

Fig. xxx Evolution of the Free Energy for the II $\leftrightarrows \mathbf{I}$ polymorph conversion reaction using temperature dependant phonon enthalpic and entropic contributions within the harmonic approximation. 
To investigate the finite temperature effect on the relative polymorphic stability associated with the II $\leftrightarrows \mathbf{I}$ equilibrium, we include the vibrational enthalpic and entropic contribution to compute the temperature dependant free energy within the harmonic approximation. The free energy is reduced by half from about $-2 \mathrm{~kJ} \cdot \mathrm{mol}^{-1}$ to about $-1 \mathrm{~kJ} \cdot \mathrm{mol}^{-1}$ between $0 \mathrm{~K}$ and room temperature, but it hardly reaches $-0.5 \mathrm{~kJ} \mathrm{~mol}^{-1}$ at $600 \mathrm{~K}$. The increase of the reaction free energy is the consequence of II having slightly more low energy vibrations that have larger entropic contributions at higher temperature than I. This could be probably linked with the slightly lower intermolecular interactions in polymoph II. Even if at high temperature, the approximation used to compute the free energy are not always accurate, it suggests that polymorph $\mathbf{I}$ is always the most stable and that stability inversion is not reached before crystal melting (in our model, stability inversion is found to be beyond $1000 \mathrm{~K}$ ). Then polymorph II is purely a kinetic product that is not stable in the accessible temperature range. We also have used the computed free energy to estimate the solubility ratio of these two polymorphs as a function of temperature (see Fig. SXX in the SI). At room temperature the solubility ratio $S_{I} S_{I I}$ is calculated to be 0.42 meaning that II is more soluble that I as II is the less stable crystal form. 


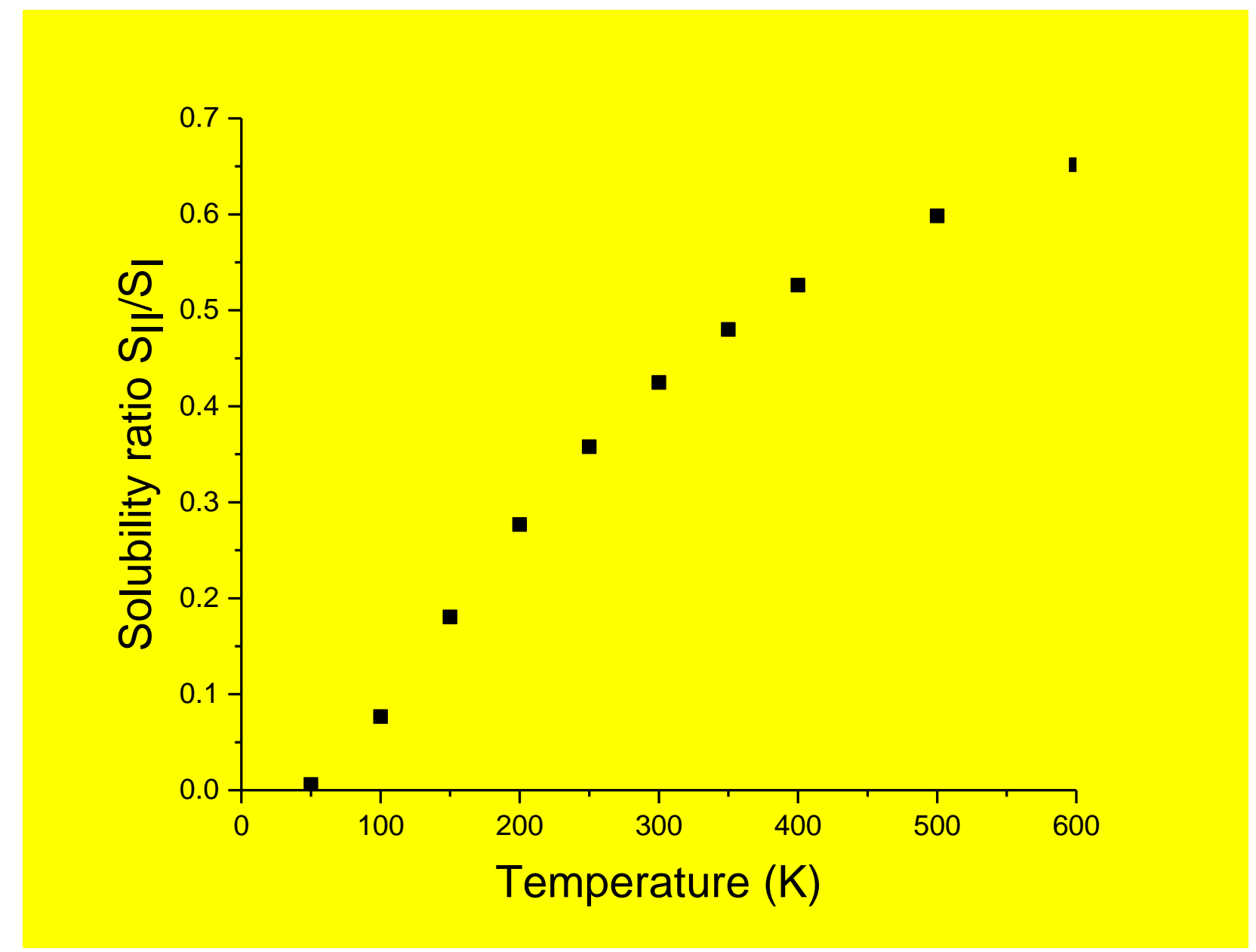

Fig Sxx: Evolution of the solubility ratio of polymorph II and I as a function of the temperature.

\section{Discussion}

A classification of polymorphs in four different types was proposed by Aitipamula et al. (1f), viz. conformational, synthon, packing and tautomeric polymorphism. As is clear from Table 1, the molecules within each polymorph and between the polymorphs are very similar, despite the torsional flexibility of the alkyl chains. The only important torsional flexibility which is observed between $\mathbf{I}$ and $\mathbf{I I}$ concern the end alcohol group which is in trans configuration for $\mathbf{I}$ and in cis for II.

It is well recognized that in the case of synthon polyphormism arising from different hydrogen networks the less stable polymorphic form is often characterized by a less likely hydrogen bond 
network than the more stable form has ${ }^{56}$ This type of polymorphism does not occur here, despite the different configurations of the alcohol groups, since the hydrogen bonding networks are very similar. This is corroborated by a hydrogen bond propensity analysis using a subset of CSD structures with acyclic aliphatic hydroxyl and cyclic thioether functional groups. ${ }^{57}$ The H-bond propensity and H-bond co-ordination scores are - not surprisingly identical - for the two observed polymorphic groups $(0.39 / 0.90)$ and more likely than any other possible network in the putative hydrogen bond network landscape, notably those involving the cyclic thioether functional group.

The ab initio DFT calculations suggest that polymorph I is slightly more stable than II. The fully optimized structure of $\mathbf{I}$ is about $150 \mathrm{meV}$ lower in energy than that of II, which corresponds to less than $1 \mathrm{meV}$ per atom. It is therefore not surprising that the two polymorphs exist. The NCI calculations and the DFT energy decompositions also suggest a slightly more stable structure for I, because of the larger accumulated charge between the herringbone stacked molecules than in II. The charge accumulated in the NCI regions in the hydrogen-bonded network is nearly the same for two polymorphs, so that the difference in stability can only be explained by the stacking mode of the molecules. The essential difference between the two polymorphic forms must thus be found in a difference between the weaker van der Waals interactions associated with the interactions between the slightly positively charged $\mathbf{C 5 O H}$ chains and the negatively charged BTBT cores.

Whereas the energy difference between the two polymorphs of BTBT-C5OH is only very small, the two individual forms have packing features that are more or less equivalent to other BTBT containing structures. The MERCURY packing similarity module was used with medium packing feature tolerances, i.e. $20^{\circ}$ at maximum for intermolecular angles and $30 \%$ for intermolecular 
distances. The BTBT cores in I are stacked in the same way as in BTBT-C2 (RMSD $=0.323 ; 2,7$ diethyl[1] benzothieno[3,2-b][1]benzothiophene, ${ }^{58}$ ), and also to a lesser extent as the cores in 2,7bis((5-methylhexyl)oxy)[1]benzothieno[3,2-b][1]benzothiophene $\quad\left(\mathrm{RMSD}=1.718 ; \quad{ }^{59}\right) . \quad$ The packing in II gives 14 hits with the same packing feature tolerances. Very close matches are found for BTBT cores symmetrically functionalized with $\mathrm{C} 8\left(\mathrm{RMSD}=0.053 ;{ }^{60}\right), \mathrm{C} 10(\mathrm{RMSD}=0.044$; $\left.{ }^{60}\right)$ and $\mathrm{C} 12\left(\mathrm{RMSD}=0.03 ;{ }^{61}\right)$ aliphatic chains. The ESI table S2 gives the complete listings of the matching structures with similar BTBT stacking patterns.

It is interesting to compare the BTBT stacking in the structure of I and II with that in the previously published symmetric structures of BTBT-C4OH ${ }^{20}$ and BTBT-C6OH ${ }^{22}$ Whilst there is no packing feature match between the BTBT cores in BTBT-C5OH-I and those in BTBTC4OH and BTBT-C6OH there is a weak match between the BTBT stacking in BTBT-C5OH-II and that in BTBT-C6OH. Fig. 8 shows the best overlay between the BTBT cores of BTBTC5OH-II (100 K) and BTBT-C6OH (95K; RMSD $=0.749$ with intermolecular angle differences between $20^{\circ}$ and $30^{\circ}$ and intermolecular distance differences between 30 and $40 \%$, thus establishing a weak packing similarity according to the MERCURY criteria). It is noted that this concerns only the stacking of the BTBT cores and not that of the complete molecules. The essential difference between the two packing features is the angle between the least-squares planes of the BTBT cores of the two structures which is acute $\left(<90^{\circ} ; 53.13^{\circ}\right)$ for BTBT-C6OH and obtuse (between 90 and $180^{\circ} ; 124.77^{\circ}$ ) for BTBT-C5OH-II (Figure 8). This angle, also called the herringbone angle determines whether there are close $S \cdots S$ contacts between neighbouring BTBT moieties or not. Obtuse interplanar angles lead to closer S $\cdots \mathrm{S}$ contacts and thus to better electronic properties than acute interplanar angles. An alternative but equivalent view is developed in Roche et al. ${ }^{30}$ where it is shown that one half of the BTBT moieties for the even members in 
the BTBT-CnOH series $(5 \leq n \leq 10)$ with herringbone-type stackings is rotated by $180^{\circ}$ with respect to the long axis of the moiety compared to the orientation in the structures of the odd members.

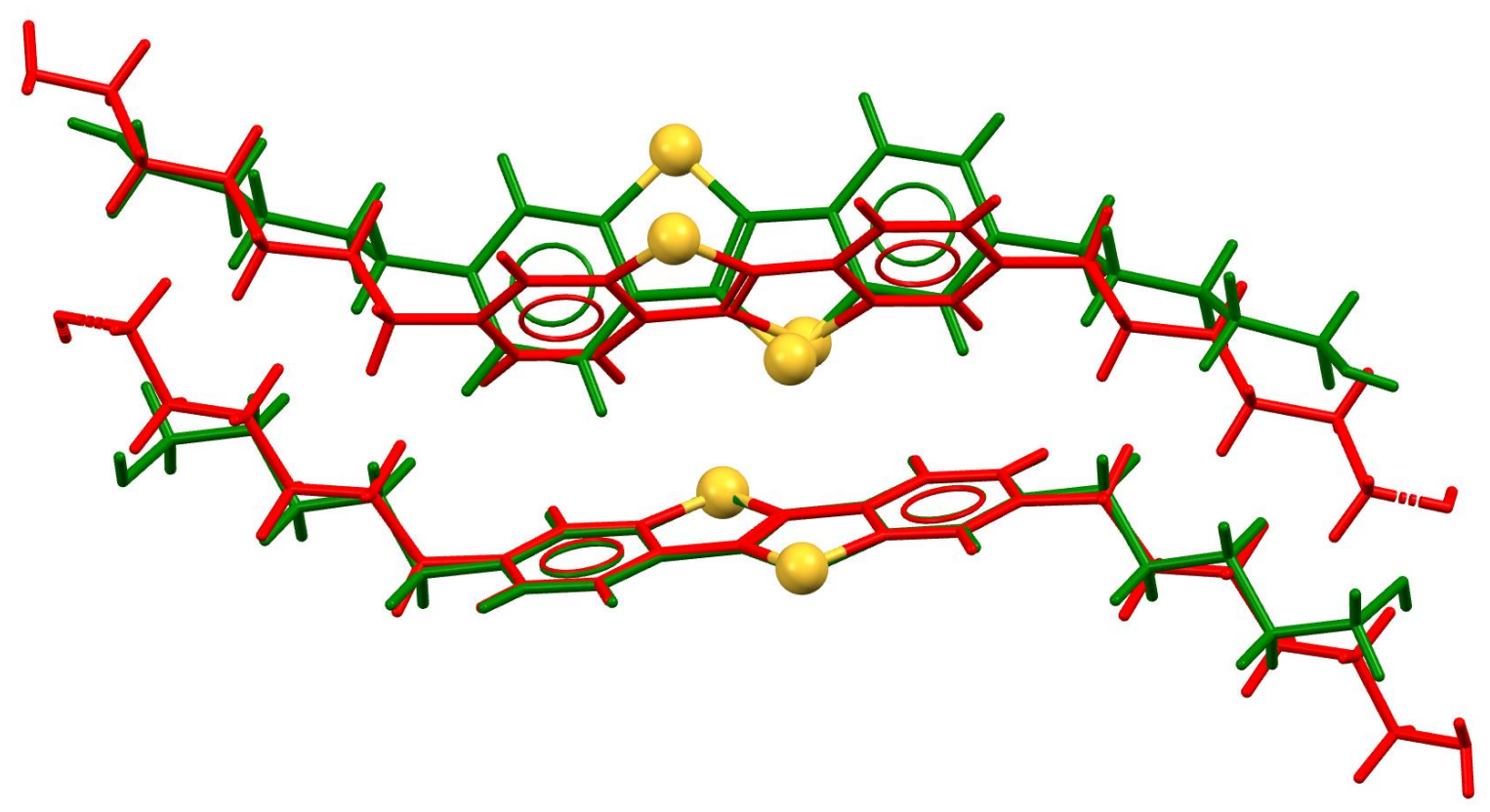

Figure 8. Packing similarity between the BTBT cores in BTBT-C5OH-II (100 K; green) and those in BTBT-C6OH (95 K; red). Both molecules are in stick representation except for the sulphur atoms. The BTBT cores of the lower pair of molecules have been aligned manually.

The average room-temperature mobility of 10 field effect transistor devices based on BTBTC5OH-II fabricated on $\mathrm{Si} / \mathrm{SIO} 2$ substrates was reported to be $0.16(2) \mathrm{cm}^{2} \mathrm{~V}^{-1} \mathrm{~s}^{-1}$ (11), which does not make it to be an excellent semiconductor, compared to e.g. BTBT-C8 $\left(1.1 \mathrm{~cm}^{2} \mathrm{~V}^{-1} \mathrm{~s}^{-1}, 40\right)$ or the organic semiconductor benchmark compound rubrene, $\mathrm{C}_{42} \mathrm{H}_{28},\left(20 \mathrm{~cm}^{2} \mathrm{~V}^{-1} \mathrm{~s}^{-1},{ }^{62}\right)$. It is known, however, that BTBT derivatives with longer side chains have in general better semiconducting properties (40). 


\section{Conclusions}

Polymorphism remains to be a badly understood phenomenon. Crystal structure prediction has

made large advances in the last decades, ${ }^{63,64}$ but the bottleneck remains the prediction of almost equally stable polymorphs and how they can be realized. ${ }^{65}$ The situation for BTBT-C5OH is not different: we are not able to say why the two polymorphs have very similar energies and why the one crystallizes in chloroform and the other in THF. A study as a function of pressure would be interesting to find out of the one form could be converted in the other. ${ }^{66,67}$ This would at the same time induce an insulator to semiconductor transition.

BTBT-C5OH clearly presents a case of packing polymorphism, although there would have been the possibility of conformational polymorphism thanks to the rotational flexibility of the terminal hydroxyl group. However, in both polymorphic forms one of the two crystallographically independent molecules has two cis orientations of the hydroxyl group and two have trans orientations. The packing polymorphism in this case can be associated with slightly different distributions of weak intermolecular interactions between the two polymorphic forms as has also been demonstrated in some other cases. ${ }^{68-70}$

The present study shows once more the importance of polymorphism in the field of BTBT based organic semiconducting and gives in particular an insight in the role of the interactions between the aliphatic side chains and the cores, which, when neglected, can lead to polymorphic structures without sufficient overlap between BTBT cores and thus to decreased charge carrier mobilities. 


\section{ASSOCIATED CONTENT}

Supporting Information. Crystal structure data of BTBT-C5OH-I and BTBT-C5OH-II, packing similarities between BTBT-C5OH-I and BTBT-C5OH-II and other structures in the Cambridge Structural Database, photomicrographs of crystals of polymorph I and II, thermal expansion data of BTBT-C5-OH-I, atomic displacement plots for the low-temperature structures of I and II with atom labelling, unusual geometric observations (Mogul analysis), 6-molecules clusters for polymorph I and II used for the promolecular NCI calculations, electron density variation between BTBT-C5-OH isolated subunits (DFT calculations). Crystallographic Information Framework (CIF) files for all structural models reported in this paper have been deposited at the Cambridge Structural Database under the numbers CCDC 2059375, 20605942060599. These data can be obtained free of charge from The Cambridge Crystallographic Data Centre via www.ccdc.cam.ac.uk/structures"

\section{AUTHOR INFORMATION}

\section{Corresponding Author}

Arie van der Lee - Institut Européen des Membranes, IEM - UMR 5635, ENSCM, CNRS, Université de Montpellier, Montpellier, France; http://orcid.org/0000-0002-4567-1831; Email : arie.van-der-lee@umontpellier.fr.

\section{Author Contributions}

The manuscript was written through contributions of all authors. All authors have given approval to the final version of the manuscript.

Notes 
The authors declare no competing financial interest.

\section{ACKNOWLEDGMENT}

GHR gratefully acknowledges financial support of ANR grant Helios N ANR-13-PRGE-0006 of the PROGELEC program. JSF acknowledges support from the French computational resource centers IDRIS and CINES (under grant 2020-A0080910369). We acknowledge the European Synchrotron Radiation Facility in Grenoble, France, for the allocation of beam time on ID29.

\section{REFERENCES}

1. Caira, M. R., Crystalline Polymorphism of Organic Compounds. In Topics in Current Chemistry, Springer Berlin Heidelberg: 1998; pp 163-208.

2. Nangia, A., Conformational Polymorphism in Organic Crystals. Accounts of Chemical Research 2008, 41 (5), 595-604.

3. Polymorphism in the Pharmaceutical Industry Edited by R. Hilfiker. WileyVCH: Weinheim. 2006. 414 + xix pp. £105. ISBN 3-527-31146-7. Organic Process Research \& Development 2007, 11 (3), 649-649.

4. Brog, J.-P.; Chanez, C.-L.; Crochet, A.; Fromm, K. M., Polymorphism, what it is and how to identify it: a systematic review. RSC Advances 2013, 3 (38), 16905.

5. Aitipamula, S.; Chow, P. S.; Tan, R. B. H., Polymorphism in cocrystals: a review and assessment of its significance. CrystEngComm 2014, 16 (17), 3451.

6. Bernstein, J., Polymorphism in Molecular Crystals. Oxford University Press: 2020.

7. Dumail, X.; Mallet-Ladeira, S.; Van der Lee, A.; Granier, D.; Masquelez, N.; Filhol, J. S.; Dutremez, S. G., Pentafluorophenylphosphonic Acid as a New Building Block for Molecular Crystal Fabrication. Cryst. Growth Design 2021.

8. Wang, C.; Xu, B.; Li, M.; Chi, Z.; Xie, Y.; Li, Q.; Li, Z., A stable tetraphenylethene derivative: aggregation-induced emission, different crystalline polymorphs, and totally different mechanoluminescence properties. Materials Horizons 2016, 3 (3), 220-225.

9. $\quad$ Sun, H.; Sun, S.-S.; Han, F.-F.; Ni, Z.-H.; Zhang, R.; Li, M.-D., A new tetraphenylethene-based Schiff base: two crystalline polymorphs exhibiting totally different photochromic and fluorescence properties. Journal of Materials Chemistry C 2019, 7 (23), 7053 7060 .

10. Láng, P.; Kiss, V.; Ambrus, R.; Farkas, G.; Szabó-Révész, P.; Aigner, Z.; Várkonyi, E., Polymorph screening of an active material. Journal of Pharmaceutical and Biomedical Analysis 2013, 84, 177-183.

11. Lee, E. H., A practical guide to pharmaceutical polymorph screening \& selection. Asian Journal of Pharmaceutical Sciences 2014, 9 (4), 163-175.

12. Yu, L., Polymorphism in Molecular Solids: An Extraordinary System of Red, Orange, and Yellow Crystals. Accounts of Chemical Research 2010, 43 (9), 1257-1266.

13. Avendano, C.; Zhang, Z.; Ota, A.; Zhao, H.; Dunbar, K. R., Dramatically Different Conductivity Properties of Metal-Organic Framework Polymorphs of Tl(TCNQ): An 
Unexpected Room-Temperature Crystal-to-Crystal Phase Transition. Angewandte Chemie International Edition 2011, 50 (29), 6543-6547.

14. Yan, D.; Evans, D. G., Molecular crystalline materials with tunable luminescent properties: from polymorphs to multi-component solids. Mater. Horiz. 2014, 1 (1), 46-57.

15. Li, X.; Ou, X.; Rong, H.; Huang, S.; Nyman, J.; Yu, L.; Lu, M., The Twelfth Solved Structure of ROY: Single Crystals of Y04 Grown from Melt Microdroplets. Crystal Growth \& Design 2020, 20 (11), 7093-7097.

16. Kersten, K.; Kaur, R.; Matzger, A., Survey and analysis of crystal polymorphism in organic structures. IUCrJ 2018, 5 (Pt 2), 124-129.

17. McCrone, W. C., In Physics and Chemistry of the Organic Solid State, Fox, D.; Labes, M. M.; Weissberger, A.; Rice, S. A., Eds. Wiley Interscience: New York, 1965; Vol. 2, pp 725767.

18. Steed, K. M.; Steed, J. W., Packing Problems: HighZ' Crystal Structures and Their Relationship to Cocrystals, Inclusion Compounds, and Polymorphism. Chemical Reviews 2015, 115 (8), 2895-2933.

19. Clegg, W., Crystallographic curiosities: polymorphism and structures with $Z^{\prime}>1$. Acta Crystallographica Section C Structural Chemistry 2019, 75 (7), 833-834.

20. Dumitrescu, D. G.; Roche, G. H.; Moreau, J. J. E.; Dautel, O. J.; van der Lee, A., A positive to negative uniaxial thermal expansion crossover in an organic benzothienobenzothiophene structure. Acta Crystallographica Section B Structural Science, Crystal Engineering and Materials 2020, 76 (4), 661-673.

21. Roche, G. H.; Tsai, Y.-T.; Clevers, S.; Thuau, D.; Castet, F.; Geerts, Y. H.; Moreau, J. J. E.; Wantz, G.; Dautel, O. J., The role of H-bonds in the solid state organization of [1]benzothieno[3,2-b][1]benzothiophene (BTBT) structures: bis(hydroxy-hexyl)-BTBT, as a functional derivative offering efficient air stable organic field effect transistors (OFETs). Journal of Materials Chemistry C 2016, 4 (28), 6742-6749.

22. van der Lee, A.; Roche, G. H.; Wantz, G.; Moreau, J. J. E.; Dautel, O. J.; Filhol, J.-S., Experimental and theoretical evidence of a supercritical-like transition in an organic semiconductor presenting colossal uniaxial negative thermal expansion. Chemical science 2018, 9 (16), 3948-3956.

23. Roche , G. H.; Bruckner , G.; Dumitrescu, D. G.; Moreau, J. J. E.; van der Lee , A.; Wantz , G.; Dautel, O. J., Structural odd-even effect impacting the dimensionality of carrier transport in BTBT-CnOH organic semiconductors. submitted 2021.

24. Jiang, H.; Yang, X.; Cui, Z.; Liu, Y.; Li, H.; Hu, W.; Liu, Y.; Zhu, D., Phase dependence of single crystalline transistors of tetrathiafulvalene. Applied Physics Letters 2007, 91 (12), 123505.

25. Käfer, D.; El Helou, M.; Gemel, C.; Witte, G., Packing of Planar Organic Molecules: Interplay of van der Waals and Electrostatic Interaction. Crystal Growth \& Design 2008, 8 (8), 3053-3057.

26. Jurchescu, O. D.; Mourey, D. A.; Subramanian, S.; Parkin, S. R.; Vogel, B. M.; Anthony, J. E.; Jackson, T. N.; Gundlach, D. J., Effects of polymorphism on charge transport in organic semiconductors. Physical Review B 2009, 80 (8).

27. Diao, Y.; Lenn, K. M.; Lee, W.-Y.; Blood-Forsythe, M. A.; Xu, J.; Mao, Y.; Kim, Y.; Reinspach, J. A.; Park, S.; Aspuru-Guzik, A.; Xue, G.; Clancy, P.; Bao, Z.; Mannsfeld, S. C. B., Understanding Polymorphism in Organic Semiconductor Thin Films through Nanoconfinement. Journal of the American Chemical Society 2014, 136 (49), 17046-17057. 
28. Hiszpanski, A. M.; Baur, R. M.; Kim, B.; Tremblay, N. J.; Nuckolls, C.; Woll, A. R.; Loo, Y.-L., Tuning Polymorphism and Orientation in Organic Semiconductor Thin Films via Post-deposition Processing. Journal of the American Chemical Society 2014, 136 (44), 1574915756.

29. Yuan, Y.; Giri, G.; Ayzner, A. L.; Zoombelt, A. P.; Mannsfeld, S. C. B.; Chen, J.; Nordlund, D.; Toney, M. F.; Huang, J.; Bao, Z., Ultra-high mobility transparent organic thin film transistors grown by an off-centre spin-coating method. Nature Communications 2014, 5 (1).

30. Roche , G. H.; Bruckner , G.; Dumitrescu, D. G.; Moreau, J. J. E.; van; der Lee , A.; Wantz , G.; Dautel, O. J., Structural odd-even effect impactin the dimensionality of carrier transport in BTBT-CnOH

organic semiconductors. JACS 2021.

31. CRYSALIS-PRO, Rigaku-Oxford Diffraction Ltd.: Yarnton, England, 2015.

32. Gabadinho, J.; Beteva, A.; Guijarro, M.; Rey-Bakaikoa, V.; Spruce, D.; Bowler, M.

W.; Brockhauser, S.; Flot, D.; Gordon, E. J.; Hall, D. R.; Lavault, B.; McCarthy, A. A.; McCarthy, J.; Mitchell, E.; Monaco, S.; Mueller-Dieckmann, C.; Nurizzo, D.; Ravelli, R. B. G.; Thibault, X.; Walsh, M. A.; Leonard, G. A.; McSweeney, S. M., MxCuBE: a synchrotron beamline control environment customized for macromolecular crystallography experiments.

Journal of synchrotron radiation 2010, 17 (5), 700-707.

33. Kabsch, W., Integration, scaling, space-group assignment and post-refinement. Acta crystallographica. Section D, Biological crystallography 2010, 66 (Pt 2), 133-144.

34. Kabsch, W., XDS. Acta crystallographica. Section D, Biological crystallography 2010, 66 (Pt 2), 125-132.

35. van der Lee, A., Charge flipping for routine structure solution. Journal of Applied Crystallography 2013, 46, 1306-1315.

36. Palatinus, L.; Chapuis, G., SUPERFLIP- a computer program for the solution of crystal structures by charge flipping in arbitrary dimensions. Journal of Applied Crystallography 2007, 40 (4), 786-790.

37. Spek, A. L., Single-crystal structure validation with the programPLATON. Journal of Applied Crystallography 2003, 36 (1), 7-13.

38. Spek, A. L., checkCIF validation ALERTS: what they mean and how to respond. Acta crystallographica. Section E, Crystallographic communications 2020, 76 (Pt 1), 1-11.

39. Betteridge, P. W.; Carruthers, J. R.; Cooper, R. I.; Prout, K.; Watkin, D. J., CRYSTALSversion 12: software for guided crystal structure analysis. Journal of Applied Crystallography 2003, 36 (6), 1487-1487.

40. Grimme, S.; Antony, J.; Ehrlich, S.; Krieg, H., A consistent and accurate ab initio parametrization of density functional dispersion correction (DFT-D) for the 94 elements $\mathrm{H}-\mathrm{Pu}$. The Journal of Chemical Physics 2010, 132 (15), 154104.

41. Kresse, G.; Joubert, D., From ultrasoft pseudopotentials to the projector augmented-wave method. Physical Review B 1999, 59 (3), 1758-1775.

42. Blöchl, P. E., Projector augmented-wave method. Physical Review B 1994, 50 (24), 17953-17979.

43. Kresse, G.; Hafner, J., Ab initiomolecular-dynamics simulation of the liquid-metalamorphous-semiconductor transition in germanium. Physical Review B 1994, 49 (20), 1425114269. 
44. Kresse, G.; Furthmüller, J., Efficiency of ab-initio total energy calculations for metals and semiconductors using a plane-wave basis set. Computational Materials Science 1996, 6 (1), $15-50$.

45. Bruno, I. J.; Cole, J. C.; Kessler, M.; Luo, J.; Motherwell, W. D. S.; Purkis, L. H.; Smith, B. R.; Taylor, R.; Cooper, R. I.; Harris, S. E.; Orpen, A. G., Retrieval of Crystallographically-Derived Molecular Geometry Information. Journal of Chemical Information and Computer Sciences 2004, 44 (6), 2133-2144.

46. Groom, C. R.; Bruno, I. J.; Lightfoot, M. P.; Ward, S. C., The Cambridge Structural Database. Acta crystallographica Section B, Structural science, crystal engineering and materials 2016, 72 (Pt 2), 171-179.

47. Macrae, C. F.; Sovago, I.; Cottrell, S. J.; Galek, P. T. A.; McCabe, P.; Pidcock, E.; Platings, M.; Shields, G. P.; Stevens, J. S.; Towler, M.; Wood, P. A., Mercury 4.0: from visualization to analysis, design and prediction. Journal of applied crystallography 2020, $53(\mathrm{Pt}$ 1), 226-235.

48. Bondi, A., van der Waals Volumes and Radii. The Journal of Physical Chemistry 1964, 68 (3), 441-451.

49. Doube, M., The Ellipsoid Factor for Quantification of Rods, Plates, and Intermediate Forms in 3D Geometries. Frontiers in endocrinology 2015, 6, 15-15.

50. Burger, A.; Ramberger, R., On the polymorphism of pharmaceuticals and other molecular crystals. II. Mikrochimica Acta 1979, 72 (3-4), 273-316.

51. Perlovich, G.; Surov, A., Polymorphism of monotropic forms: relationships between thermochemical and structural characteristics. Acta Crystallographica Section B Structural Science, Crystal Engineering and Materials 2020, 76 (1), 65-75.

52. van de Streek, J.; Neumann, M. A., Validation of experimental molecular crystal structures with dispersion-corrected density functional theory calculations. Acta crystallographica. Section B, Structural science 2010, 66 (Pt 5), 544-558.

53. Bergerhoff, G.; Berndt, M.; Brandenburg, K.; Degen, T., Concerning inorganic crystal structure types. Acta Crystallographica Section B Structural Science 1999, 55 (2), 147-156.

54. de la Flor, G.; Orobengoa, D.; Tasci, E.; Perez-Mato, J. M.; Aroyo, M. I., Comparison of structures applying the tools available at the Bilbao Crystallographic Server. Journal of Applied Crystallography 2016, 49 (2), 653-664.

55. Otero-de-la-Roza, A.; Johnson, E.; Contreras-Garcia, J., Revealing non-covalent interactions in solids: NCI plots revisited. Physical Chemistry Chemical Physics 2012, 14 (35), 12165-12172.

56. Galek, P. T. A.; Allen, F. H.; Fábián, L.; Feeder, N., Knowledge-based H-bond prediction to aid experimental polymorph screening. CrystEngComm 2009, 11 (12), 2634.

57. Galek, P. T. A.; Fábián, L.; Motherwell, W. D. S.; Allen, F. H.; Feeder, N., Knowledgebased model of hydrogen-bonding propensity in organic crystals. Acta Crystallographica Section B Structural Science 2007, 63 (5), 768-782.

58. Minemawari, H.; Tanaka, M.; Tsuzuki, S.; Inoue, S.; Yamada, T.; Kumai, R.; Shimoi, Y.; Hasegawa, T., Enhanced Layered-Herringbone Packing due to Long Alkyl Chain Substitution in Solution-Processable Organic Semiconductors. Chemistry of Materials 2017, 29 (3), 1245-1254.

59. Ruzié, C.; Karpinska, J.; Laurent, A.; Sanguinet, L.; Hunter, S.; Anthopoulos, T. D.; Lemaur, V.; Cornil, J.; Kennedy, A. R.; Fenwick, O.; Samorì, P.; Schweicher, G.; Chattopadhyay, B.; Geerts, Y. H., Design, synthesis, chemical stability, packing, cyclic 
voltammetry, ionisation potential, and charge transport of [1]benzothieno[3,2-

b][1]benzothiophene derivatives. Journal of Materials Chemistry C 2016, 4 (22), 4863-4879.

60. Izawa, T.; Miyazaki, E.; Takimiya, K., Molecular Ordering of High-Performance

Soluble Molecular Semiconductors and Re-evaluation of Their Field-Effect Transistor

Characteristics. Advanced Materials 2008, 20 (18), 3388-3392.

61. Ebata, H.; Izawa, T.; Miyazaki, E.; Takimiya, K.; Ikeda, M.; Kuwabara, H.; Yui, T., Highly Soluble [1]Benzothieno[3,2-b]benzothiophene (BTBT) Derivatives for High-

Performance, Solution-Processed Organic Field-Effect Transistors. Journal of the American Chemical Society 2007, 129 (51), 15732-15733.

62. Sundar, V. C., Elastomeric Transistor Stamps: Reversible Probing of Charge Transport in Organic Crystals. Science 2004, 303 (5664), 1644-1646.

63. Graser, J.; Kauwe, S. K.; Sparks, T. D., Machine Learning and Energy Minimization Approaches for Crystal Structure Predictions: A Review and New Horizons. Chemistry of Materials 2018, 30 (11), 3601-3612.

64. Oganov, A. R.; Pickard, C. J.; Zhu, Q.; Needs, R. J., Structure prediction drives materials discovery. Nature Reviews Materials 2019, 4 (5), 331-348.

65. Cruz-Cabeza, A. J., Crystal structure prediction: are we there yet? Acta Crystallographica Section B Structural Science, Crystal Engineering and Materials 2016, 72 (4), 437-438.

66. Zakharov, B. A.; Tumanov, N. A.; Boldyreva, E. V., $\beta$-Alanine under pressure: towards understanding the nature of phase transitions. CrystEngComm 2015, 17 (10), 2074-2079.

67. Fedorov, A. Y.; Rychkov, D. A.; Losev, E. A.; Zakharov, B. A.; Stare, J.; Boldyreva, E. V., Effect of pressure on two polymorphs of tolazamide: why no interconversion?

CrystEngComm 2017, 19 (16), 2243-2252.

68. Kulkarni, G. U.; Kumaradhas, P.; Rao, C. N. R., Charge Density Study of the Polymorphs ofp-Nitrophenol. Chemistry of Materials 1998, 10 (11), 3498-3505.

69. Surov, A. O.; Manin, A. N.; Voronin, A. P.; Churakov, A. V.; Perlovich, G. L.; Vener, M. V., Weak Interactions Cause Packing Polymorphism in Pharmaceutical Two-Component Crystals. The Case Study of the Salicylamide Cocrystal. Crystal Growth \& Design 2017, 17 (3), 1425-1437.

70. Karmakar, A.; Hazra, S.; Rúbio, G. M. D. M.; Guedes da Silva, M. F. C.; Pombeiro, A. J. L., Packing polymorphism in 3-amino-2-pyrazinecarboxylate based tin(ii) complexes and their catalytic activity towards cyanosilylation of aldehydes. New Journal of Chemistry 2018, 42 (21), 17513-17523.

Figure for Table of Contents 


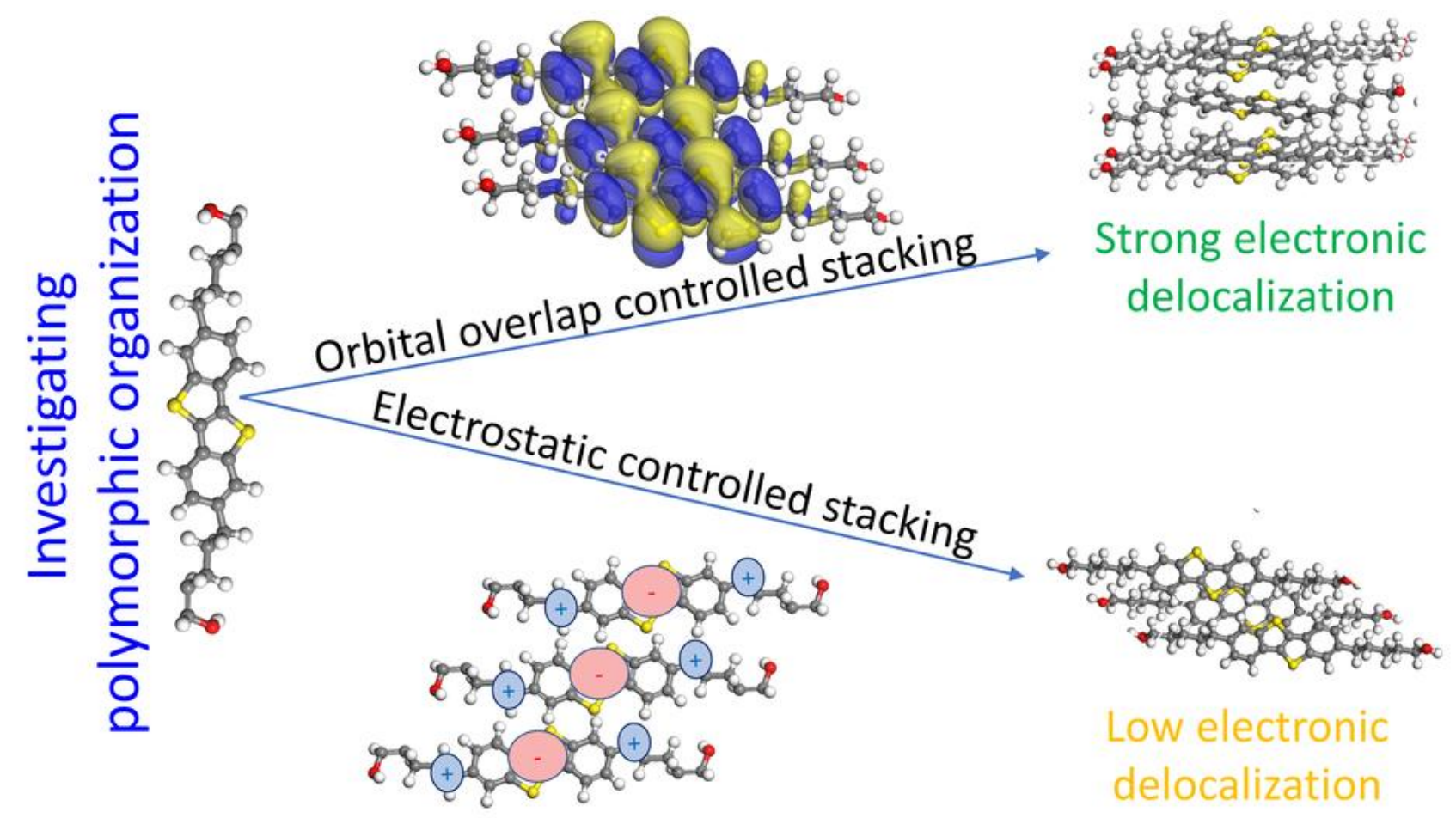

